\title{
HOLOMORPHIC EXTENSION AND DECOMPOSITION FROM A TOTALLY REAL MANIFOLD
}

\author{
ZAIFEI YE
}

\begin{abstract}
This paper is to develop an elementary cohomological approach for decomposing a function into boundary values of holomorphic functions and for discussing the corresponding microlocal analysis and hyperfunction theory.
\end{abstract}

\section{INTRODUCTION}

This work can be viewed as a residue approach to the holomorphic extension and holomorphic decomposition of functions on a totally real manifold $M \subset$ $\mathrm{C}^{m}$.

The idea is the following. To a compactly supported function (distribution, analytic functional or hyperfunction) $f$ on $M$, we assign a $\bar{\partial}$ closed $(0, m-1)$ form $G$ in $\mathbf{C}^{m} \backslash M$ by a duality formula:

$$
\int_{\partial U} G \wedge \psi d z=\int_{M} f \psi d z
$$

where $U$ is a neighborhood of $\operatorname{supp}(f)$ in $\mathbf{C}^{m}, \psi$ is any holomorphic function in $\mathbf{C}^{m}$. Such a form $G$ will be called a Tsuno representation form in the paper (see $\$ 4.1$ ). Presumably, any information about $f$ is already hidden in $G$ since $G$ is in fact a kind of potential created by $f$. We then investigate the form $G$ to obtain the decomposition of $f$ into a sum of boundary values of holomorphic functions in certain wedges under various smoothness requirements, and to deal with the analytic wavefront set of $f$. The method is basically to view the $\bar{\partial}$ closed form $G$ as an element in the Dolbeault cohomology group, and to translate $G$ to a holomorphic cocycle in the corresponding Cech cohomology group. The resulting cocycle will be our holomorphic decomposition in some sense. Now, the point is to have a very concrete version of the translation from the Dolbeault cohomology to the Čech cohomology. This can be done by solving a sequence of $\bar{\partial}$ problems (so called "Weil process"). In this paper, we only use the elementary part of some techniques in several complex variables such as $\bar{\partial}$ solutions and the Bochner integral representation.

To deal with a curved edge instead of a straight edge, one has to replace "suitably" the covering of $\mathbf{C}^{m} \backslash \mathbf{R}^{m}$ given by $\left\{x_{j}>\right.$ (or $<$ ) 0$\}$. This is a matter

Received by the editors December 4, 1990 and, in revised form, June 21, 1991.

1991 Mathematics Subject Classification. Primary 32D15, 32A07.

Key words and phrases. Holomorphic functions, boundary values, totally real manifold, wave front sets, analytic functionals, $\bar{\partial}$ problems. 
which does not seem to have been well exploited previously (e.g., in the proof of the edge-of-the-wedge theorem, Pincuk has a more difficult approach to keep the cohomological approach of Martineau). In this setting, it is almost inevitable to raise the question of the existence of the boundary values of holomorphic functions without growth condition on a (non-real-analytic) edge, i.e. the existence in the hyperfunction sense.

We have proved: (i) the existence of the boundary values of holomorphic functions in wedges on a $C^{1}$ totally real edge in the hyperfunction sense (\$3.2); (ii) the decomposition of any analytic functional supported on $M$ into a sum of boundary values of holomorphic functions in wedges ( $\$ 4.2$ ); (iii) a characterization of the wave front set in terms of the $\bar{\partial}$ exactness of $G$ in certain concave wedge ( $\S 5.2$ ); (iv) the edge-of-the-wedge theorem with a $C^{2}$ totally real edge in the hyperfunction sense ( $\$ 5.3)$.

We give some new proofs and slight extensions of certain fundamental results based on the above ideas. We first precise some properties of the holomorphic decomposition of the Baouendi-Chang-Treves $\left(C^{\infty}\right.$ decomposition $)$ as an illustration of the residue approach in the paper (see $\S 2.2)$. We then investigate the relation between the holomorphic extension to wedges and some phenomenon of monodromy of differential forms $(\S 5.3)$. We also study the flabbiness properties of the sheaf of germs of hyperfunctions on a $C^{1}$ totally real manifold and $\bar{\partial}$ problem with partial compact support in $\S 6$.

In addition, we append an elementary proof of Pincuk's edge-of-the-wedge theorem for distributions by using the Baouendi-Treves approximation technique, which is totally independent.

The idea in the paper can be traced back to the Cauchy integral and Plemelj's jump formula. Namely, when considering a function $f$ compactly supported on a curve $M$ in $\mathbf{C}^{1}$, we put

$$
G(\zeta)=\frac{1}{2 \pi i} \int_{M} \frac{f(z)}{z-\zeta} d z .
$$

It is clear that $G$ is holomorphic in $\mathbf{C}^{1} \backslash \operatorname{supp}(f)$ and satisfies

$$
\int_{\partial U} G \psi=\int_{M} f \psi
$$

for any neighborhood $U$ of $\operatorname{supp}(f)$ in $\mathbf{C}^{1}$, and any entire function $\psi$. Then, by Plemelj's formula, we know $f$ is the jump of $G$ on $M$.

As a remark, let us note that such an idea has an impact on the holomorphic decomposition of $\mathrm{CR}$ functions on hypersurfaces and higher codimensional CR manifolds (see [HvPo] and [Am]). Therefore, the residue approach in the paper may shed some light on the decomposition problems of CR functions.

As is well known, there is another very different method to deal with the decomposition problem, namely, the Fourier transform method. In $\S 5.4$, we will discuss the FBI transform of a hyperfunction. We feel that we are trying to have a synthesis of the hyperfunction theory of Sato-Kawai-Kashiwara (and Martineau) and the FBI transform method. The treatment of the hyperfunction theory in Hörmander's book [H1] has been enlightening our work.

The point of view in the paper is very local except for $\S 6$. The unpleasant part of the paper is that the smoothness requirement varies from section to section. Here, for convenience, we list the requirements: (i) $C^{\infty}$ for the case 
where distributions or smooth functions are involved ( $\S 2,6.2$, and Appendix); (ii) $C^{2}$ for $\S \S 4.2$ and 5 where the Stein coverings or the FBI transforms are needed; (iii) $C^{1}$ for the rest.

The plan of the paper is the following. We begin with a description of the concrete translation from the Dolbeault cohomology to the Čech cohomology in $\S 2.1$, and then show the reader immediately how to decompose a smooth function into a sum of the smooth boundary values of holomorphic functions defined in wedges in $\S 2.2$. Sections 3 and 4 are devoted to the decomposition of a hyperfunction into the boundary values of holomorphic functions. Especially, we introduce the Tsuno representation forms and study their behaviors in $\S 4.1$. In $\S 5$, we study the (hypo) analytic wave front set according to the Tsuno forms and also to the FBI transforms. In $\S 6$, we deal with the flabbiness of the sheaf of germs of hyperfunctions, relation between distribution boundary values and hyperfunction boundary values, decomposition of a distribution into boundary values of holomorphic functions (a ne: $\mathrm{w}$ proof of the Baouendi-Chang-Treves theorem), and a $\bar{\partial}$ problem with partial compact support in some cylindrical domains. In particular, we provide a Greiner-Kohn-Stein condition for such a problem.

\section{2. $\bar{\partial}$ TECHNIQUES AND HOLOMORPHIC DECOMPOSITION IN THE SMOOTH SENSE}

2.1. $\bar{\partial}$ techniques and results to be used. The related $\bar{\partial}$ problems in the paper arises from the isomorphism (so called "Weil process") between the Dolbeault cohomology and the Čech cohomology. Before describing this process, we introduce some notations.

In this section, $M$ denotes a $C^{\infty}$ totally real manifold in $\mathbf{C}^{m}$ of real dimension $m$. Assume $0 \in M$. Let $\tilde{\rho}_{1}, \ldots, \tilde{\rho}_{m} \in C^{\infty}(\Omega), \Omega$ a neighborhood of 0 in $\mathbf{C}^{m}$, be defining functions of $M$, i.e., $\tilde{\rho}_{1}=\cdots=\tilde{\rho}_{m}=0, \bar{\partial} \tilde{\rho}_{1} \wedge \cdots \wedge \bar{\partial} \tilde{\rho}_{m} \neq 0$ on $M$. Choose $c$ sufficiently large, then it is easy to check that

$$
\rho_{j}=: \begin{cases}-\tilde{\rho}_{j}+c \chi, & j=1, \ldots, m, \\ \tilde{\rho}_{-j}+c \chi, & j=-1, \ldots,-m,\end{cases}
$$

are strictly plurisubharmonic on $\Omega$ if $\Omega$ is a small enough neighborhood of 0 , where $\chi=\sum_{j=1}^{m} \tilde{\rho}_{j}^{2}$. Thus, if one takes $\Omega$ to be strongly pseudoconvex,

$$
U_{j}=:\left\{z \in \Omega: \rho_{j}(z)<0\right\}, \quad j= \pm 1, \ldots, \pm m,
$$

are domains of holomorphy (in fact, intersections of strongly pseudoconvex domains). Moreover, $\bar{U}_{j} \cap \bar{U}_{-j}=M$, and $\mathscr{U}=\left\{U_{j}\right\}_{j= \pm 1, \ldots, \pm m}$ is a Stein covering of the set $\Omega \backslash M$. Note that, for the existence of such a Stein covering of $\Omega \backslash M$, it suffices to assume $M$ is $C^{2}$ smooth.

Weil process induces the isomorphism between the Dolbeault cohomology group $H^{0, m-1}(\Omega \backslash M)$ and the Čech cohomology group $\check{H}^{m-1}(\mathscr{U}, \mathscr{O})$, where $\mathscr{O}$ is the sheaf of holomorphic functions on $\Omega \backslash M$. Starting with a cocycle in the cochain group $C^{m-1}(\mathscr{U}, \mathscr{O})$, we come up with a $\bar{\partial}$ closed $(0, m-1)$ form (see, for example, $[\mathrm{P}])$. As a matter of fact, the reverse process is just simple to explain from the viewpoint of $\bar{\partial}$ technique.

Denote $U_{i_{1} i_{2} \ldots i_{k}}=U_{i_{1}} \cap U_{i_{2}} \cap \cdots \cap U_{i_{k}}, i_{p} \in\{ \pm 1, \ldots, \pm m\}, p=1, \ldots, k$. Let $G$ be a $\frac{\partial}{\partial}$ closed $(0, m-1)$ form defined on $\Omega \backslash M$ (later it will be the 
Tsuno representation forms). Put $G_{i}=\left.G\right|_{U_{i}}, i= \pm 1, \ldots, \pm m$. Since $U_{i}$ is pseudoconvex, there is a $(0, m-2)$ form $F_{i}$ so that $\bar{\partial} F_{i}=G_{i}$ on $U_{i}$. Hence, $G_{i j}=: F_{j}-F_{i}$ is a $\bar{\partial}$ closed $(0, m-2)$ form defined on $U_{i j}$ which is still pseudoconvex. Inductively, suppose we have a $\bar{\partial}$ closed $(0, m-q)$ form $G_{i_{1} \cdots i_{q}}$ defined on $U_{i_{1} \cdots i_{q}}$, then again, there is $F_{i_{1} \cdots i_{q}}$, a $(0, m-q-1)$ form on $U_{i_{1} \cdots i_{q}}$, so that $\bar{\partial} F_{i_{1} \cdots i_{q}}=G_{i_{1} \cdots i_{q}}$ if $q<m$. It is easy to check that

$$
G_{i_{1} \cdots i_{q+1}}=: \sum_{p=1}^{q+1}(-1)^{p-1} F_{i_{1} \cdots \hat{i}_{p} \cdots i_{q+1}}
$$

is a $\bar{\partial}$ closed $(0, m-q-1)$ form defined on $U_{i_{1} \cdots i_{q} i_{q+1}}$, where $\hat{i}_{p}$ stands for the index omitted. Finally, $G_{i_{1} \cdots i_{m}},\left|i_{1}\right|<\cdots<\left|i_{m}\right|$, is a holomorphic function defined on $U_{i_{1} \cdots i_{m}} .\left\{G_{i_{1} \cdots i_{m}},\left|i_{1}\right|<\cdots<\left|i_{m}\right|\right\}$ is a cocycle in $C^{m-1}(\mathscr{U}, \mathscr{O})$.

Therefore, we need to solve $\bar{\partial}$ problem in the intersections of the strictly pseudoconvex domains. To catch more precise information for the later purpose, we also need the following a priori estimates for the $\bar{\partial}$ solutions.

Let $\Delta$ be an open set in $\mathbf{C}^{m}$, and let $\phi$ be a function on $\Delta$. We denote by $L^{2}(\Delta, \phi)$ the Hilbert space of all functions $f$ satisfying $\int_{\Delta}|f|^{2} e^{-\phi}<\infty$, and by $L_{(p, q)}^{2}(\Delta, \phi)$ the space of all $(p, q)$ forms with coefficients in $L^{2}(\Delta, \phi)$.

Theorem 2.1.1 [H2, Theorem 2.2.3]. Let $\Delta$ be a bounded pseudoconvex open set in $\mathbf{C}^{m}$, let $\delta$ be the diameter of $\Delta$, and let $\phi$ be a plurisubharmonic function in $\Delta$. For every $f \in L_{(p, q)}^{2}(\Delta, \phi), q>0$, with $\bar{\partial} f=0$, one can find $u \in$ $L_{(p, q-1)}^{2}(\Delta, \phi)$ so that $\bar{\partial} u=f$ and

$$
q \int_{\Delta}|u|^{2} e^{-\phi} \leq e \delta^{2} \int_{\Delta}|f|^{2} e^{-\phi}
$$

The next result takes care of the smoothness of the $\bar{\partial}$ solutions. It is a typical case of the main theorem in [Duf].

Theorem 2.1.2. Let $\Delta$ be an intersection of a finite number of strictly pseudoconvex domains. If $f$ is $a(p, q)$ form, $q>0$, with coefficients in $C^{\infty}(\bar{\Delta})$, then there is $a(p, q-1)$ form $u$ with coefficients also in $C^{\infty}(\bar{\Delta})$ so that $\bar{\partial} u=f$.

2.2. Holomorphic decomposition in the smooth sense. To see the residue method in the paper, we begin with proving the $C^{\infty}$ version of the BaouendiChang-Treves decomposition theorem [BCT], which can still be proved by the FBI transform method though (see [Cal]). Our proof is a generalization of a technique developed by Amar [Am], which enables us to take care of the smoothness of $f$ in terms of its Tsuno representation form $G$ in (1.1). It is worthwhile to notice that one can use the argument below for the decomposition of $\mathrm{CR}$ functions in higher codimension case as Amar did in the codimension 2 case.

Theorem 2.2.1. Let $f$ be a smooth function on $M$, then there are holomorphic functions $h_{j_{1} \cdots j_{m}}$ defined respectively in $U_{j_{1} \cdots j_{m}},\left|j_{1}\right|<\cdots<\left|j_{m}\right|$, and smooth up to the edge $M$ so that

$$
f=\left.\sum_{\substack{j_{p}= \pm p \\ p=1, \ldots, m}} h_{j_{1} \cdots j_{m}}\right|_{M} .
$$


Proof. (1) We first construct a Tsuno representation form $G$ of $f$. Extend $f$ smoothly to $\Omega$, a neighborhood of 0 as defined before, so that $\bar{\partial} f$ vanishes on $M$ to the infinite order by Whitney's extension theorem. Choose a bounded function $\chi_{j} \in C^{\infty}(\Omega \backslash M)$ so that

$$
\chi_{j}\left|U_{j}=1, \quad \chi_{j}\right| U_{-j}=0, \quad j=1, \ldots, m .
$$

Note that $\bar{U}_{j} \cap \bar{U}_{-j}=M$ and $\operatorname{dist}\left(z, \partial U_{j}\right)+\operatorname{dist}\left(z, \partial U_{-j}\right) \cong \operatorname{dist}(z, M)^{2}$, thus, we can further choose $\chi_{j}$ so that its derivatives have polynomial growth near $M$ and $\bar{\partial} \chi_{j}$ has singularities only on $M$. Since $\bar{\partial} f$ vanishes on $M$, the $(0, m)$ current $-\chi_{1} \bar{\partial} f \wedge \bar{\partial} \chi_{2} \wedge \cdots \wedge \bar{\partial} \chi_{m}$ extends smoothly to $\bar{\Omega}$. It is $\bar{\partial}$ closed (top degree), and hence, there exists a smooth $(0, m-1)$ form $\widetilde{G}$ in $\bar{\Omega}$ satisfying

$$
\bar{\partial} \widetilde{G}=-\chi_{1} \bar{\partial} f \wedge \bar{\partial} \chi_{2} \wedge \cdots \wedge \bar{\partial} \chi_{m}
$$

Put

$$
G=f \chi_{1} \bar{\partial} \chi_{2} \wedge \cdots \wedge \bar{\partial} \chi_{m}+\tilde{G}
$$

We have therefore constructed a $(0, m-1)$ form $G$ which is $\bar{\partial}$ closed in $\Omega \backslash M$ (and hence, an element in $H^{(0, m-1)}(\Omega \backslash M)$ ), and such that

$$
G-f \chi_{1} \bar{\partial} \chi_{2} \wedge \cdots \wedge \bar{\partial} \chi_{m} \text { is smooth. }
$$

Note. (i) Although this is not needed in the proof, it should be pointed out that, at least if we consider a compactly supported data $f$, the form $G$ is a Tsuno form for $f$ (see Remark 2.2.2 below). Condition (2.4) is the one which imposes the right singularity of $G$ (as condition (b) below does later).

(ii) The next step is a common feature in our decomposition technique for smooth function, distribution and analytic functionals, namely, trying to make use of the Weil process. The only difference is that we need to solve out certain special $\bar{\partial}$ solutions for different smoothness requirements. Following the previous notations, we are going to obtain $G_{j_{1} \cdots j_{m}}$ smooth up to the boundary of $U_{j_{1} \cdots j_{m}}$ by induction, which will be our holomorphic decomposition of $f$ except for some signatures.

Let us continue to prove the theorem.

(2) Set $G_{j}=\left.G\right|_{U_{j}}$. Note that on $\bar{U}_{j}, \chi_{1} \bar{\partial} \chi_{2} \wedge \cdots \wedge \bar{\partial} \chi_{m}=0$, unless $j=1$, hence $G_{j}$ is smooth in view of (2.3).

(i) For $j \neq 1$, then, there exists $F_{j} \in C_{(0, m-2)}^{\infty}\left(\bar{U}_{j}\right)$ so that $\bar{\partial} F_{j}=G_{j}, j \neq 1$, by Theorem 2.1.2.

(ii) On $\bar{U}_{1}$, the current $\chi_{2} \bar{\partial} f \wedge \bar{\partial} \chi_{3} \wedge \cdots \wedge \bar{\partial} \chi_{m}$ is smooth, hence $G-$ $\bar{\partial}\left(f \chi_{2} \bar{\partial} \chi_{3} \wedge \cdots \wedge \bar{\partial} \chi_{m}\right)$ is smooth. This allows us to find $F_{1}$ so that

$$
F_{1}-f \chi_{2} \bar{\partial} \chi_{3} \wedge \cdots \wedge \bar{\partial} \chi_{m} \text { is smooth. }
$$

and $\bar{\partial} F_{1}=G_{1}$.

(3) Inductively, assume that we have constructed a $(p-1)$ cochain of $(0, m-$ $1-p)$ forms $F_{j_{1} \cdots j_{p}}, j_{q} \in\{ \pm 1, \ldots, \pm m\},\left|j_{q}\right|<\left|j_{q+1}\right|, q=1, \ldots, p$ so that with the following properties

(a) $F_{j_{1} \cdots j_{p}}$ is smooth on $\bar{U}_{j_{1} \cdots j_{p}}$ for $\left(j_{1} \cdots j_{p}\right) \neq(1, \ldots, p)$;

(b) $F_{1 \ldots p}-(-1)^{(p-1) p / 2} f \chi_{p+1} \bar{\partial} \chi_{p+2} \wedge \cdots \wedge \bar{\partial} \chi_{m}$ is smooth on $\bar{U}_{1 \cdots p}$;

(c) $\bar{\partial} F_{j_{1} \cdots j_{p}}=G_{j_{1} \cdots j_{p}}$ on $U_{j_{1} \cdots j_{p}}$ where $G_{j_{1} \cdots j_{p}}=\sum_{q=1}^{p}(-1)^{q+1} F_{j_{1} \cdots \hat{j}_{q} \cdots j_{p}}$. 
Then, we obtain a $\bar{\partial}$ closed $(0, m-p-1)$ form $G_{j_{1} \cdots j_{p+1}}$ on $U_{j_{1} \cdots j_{p+1}}$ defined as above. Note that (b) implies that $F_{1 \ldots p}$ is smooth on $\bar{U}_{1 \ldots p, l}$ unless $l=p+1$. And on $\bar{U}_{1, \ldots, p, p+1},(\mathrm{~b})$ yields that $F_{1 \cdots p}-(-1)^{(p-1) p / 2} \bar{\partial}\left(f \chi_{p+2} \bar{\partial} \chi_{p+3} \wedge \cdots \wedge \bar{\partial} \chi_{m}\right)$ is smooth since $\bar{\partial} f$ vanishes to $\infty$ order along $M$. In this case,

$$
\begin{gathered}
G_{1 \cdots p+1}-\bar{\partial}\left((-1)^{p(p+1) / 2} f \chi_{p+2} \bar{\partial} \chi_{p+3} \wedge \cdots \wedge \bar{\partial} \chi_{m}\right)=\sum_{q=1}^{p}(-1)^{q+1} F_{1 \cdots \hat{q} \cdots p+1} \\
+(-1)^{p+2}\left(F_{1 \cdots p+1}-\bar{\partial}\left((-1)^{p(p-1) / 2} f \chi_{p+2} \bar{\partial} \chi_{p+3} \wedge \cdots \wedge \bar{\partial} \chi_{m}\right)\right)
\end{gathered}
$$

is smooth on $\bar{U}_{1, \ldots, p+1}$ and $\bar{\partial}$ closed since $G_{1, \ldots, p+1}$ is $\bar{\partial}$ closed as we have seen in the previous section. Therefore, we see the following:

$\left(\mathbf{a}^{\prime}\right)$ when $\left\{j_{1}, \ldots, j_{p+1}\right\} \neq\{1, \ldots, p+1\}, G_{j_{1} \cdots j_{p+1}} \in C_{(0, m-p-1)}^{\infty}\left(\bar{U}_{j_{1} \cdots j_{p+1}}\right)$, and consequently, there is $F_{j_{1} \cdots j_{p+1}} \in C^{\infty}\left(\bar{U}_{j_{1} \cdots j_{p+1}}\right)$ so that

$$
\bar{\partial} F_{j_{1} \cdots j_{p+1}}=G_{j_{1} \cdots j_{p+1}} \text {; }
$$

$\left(\mathrm{b}^{\prime}\right)$ on $\bar{U}_{1 \ldots p+1}$, there is a smooth form $F_{1, \ldots, p+1}$ so that

$$
F_{1 \cdots p+1}-(-1)^{p(p+1) / 2} f \chi_{p+2} \bar{\partial} \chi_{p+3} \wedge \cdots \wedge \bar{\partial} \chi_{m} \text { is smooth, }
$$

and

$$
\bar{\partial} F_{1, \ldots, p+1}=G_{1, \ldots, p+1} .
$$

(4) Finally, this leads to a $(m-2)$ cochain of functions ( 0 forms) such that $1\}$;

$\left(\mathbf{a}^{\prime \prime}\right) F_{j_{1} \cdots j_{m-1}}$ is smooth on $\bar{U}_{j_{1} \cdots j_{m-1}}$ unless $\left\{j_{1}, \ldots, j_{m-1}\right\}=\{1, \ldots, m-$

(b') $F_{1 \ldots m-1}-(-1)^{(m-2)(m-1) / 2} f \chi_{m}$ is smooth on $\bar{U}_{1, \ldots, m-1}$;

(c) $\bar{\partial} F_{j_{1} \cdots j_{m-1}}=G_{j_{1} \cdots j_{m-1}}$ (where $G_{j_{1} \cdots j_{m-1}}$ is the coboundary defined in (c)).

Set $G_{j_{1} \cdots j_{m}}=\sum_{q=1}^{m}(-1)^{q+1} F_{j_{1} \cdots \hat{j}_{q} \cdots j_{m}}$ as before. Then, $\bar{\partial} G_{j_{1} \cdots j_{m}}=0$ (coboundary of coboundary!), i.e., $G_{j_{1} \cdots j_{m}}$ is holomorphic. Furthermore, $G_{j_{1} \cdots j_{m}}$ are smooth up to $M$ from $\left(\mathrm{a}^{\prime \prime}\right)$, $\left(\mathrm{b}^{\prime \prime}\right)$, and we assert

$$
f=\left.(-1)^{(m-1) m / 2} \sum_{\substack{j_{q}= \pm q \\ q=1, \ldots, m}} \varepsilon_{j_{1} \cdots j_{m}} G_{j_{1} \cdots j_{m}}\right|_{M} .
$$

where $\varepsilon_{j_{1} \cdots j_{m}}=+1$ if $j_{1} \cdots j_{m}>0, \varepsilon_{j_{1} \cdots j_{m}}=-1$ if $j_{1} \cdots j_{m}<0$.

Indeed, on $M$,

$$
\begin{aligned}
& \sum_{\substack{j_{q}= \pm q \\
q=1, \ldots, m}} \varepsilon_{j_{1} \cdots j_{m}} G_{j_{1} \cdots j_{m}} \\
& =\sum \sum_{\substack{\left(j_{1}, \ldots, j_{m}\right) \neq(1, \ldots, m-1, \pm m) \\
\text { or } q \neq m}}(-1)^{q+1} \varepsilon_{j_{1} \cdots j_{m}} F_{j_{1} \cdots \hat{j}_{q} \cdots j_{m}}+(-1)^{m+1}(\alpha-\beta),
\end{aligned}
$$

where $\alpha$ and $\beta$ denote the smooth extensions of the restrictions of $F_{1, \ldots, m-1}$ to $U_{1 \cdots m}$ and $U_{1, \ldots, m-1,-m}$ respectively. Due to $\left(\mathbf{b}^{\prime \prime}\right), \alpha-\beta=(-1)^{(m-2)(m-1) / 2} f$. So, due to the obvious cancellation between

$$
\varepsilon_{j_{1} \cdots j_{q} \cdots j_{m}} F_{j_{1} \cdots \hat{j}_{q} \cdots j_{m}} \text { and } \varepsilon_{j_{1} \ldots,-j_{q}, \ldots, j_{m}} F_{j_{1}, \ldots, \hat{-j_{q}}, \ldots, j_{m}},
$$

the above sum is reduced to $(-1)^{(m-1) m / 2} f$. 
(2.5) is the decomposition which we look for.

Note the fact that $\alpha \neq \beta$ is the whole point. This is why one does not get 0 . Everything else cancels out essentially due to the fact that the sum of boundary values of coboundary is 0 .

Remark 2.2.1. Notice that the smoothness assumption in the theorem can be weakened to $C^{k}$ for sufficiently large $k$. In this case, all the currents involved in the above proof make sense. Thus, Range and Siu's uniform estimate of $\bar{\partial}$ solutions on the intersections of strongly pseudoconvex domains [RSi] shows that we can obtain $G_{j_{1} \cdots j_{m}}$ continuous up to the edge $M$ and the decomposition relation (2.5) remains valid. Furthermore, by a regularization argument employed in [T, Theorem 3.1, Chapter II], one can obtain the decomposition theorem in the distribution sense, which is proved in [BCT] with FBI transform. In fact, one can choose certain vector fields $M_{j}$ on $M, j=1, \ldots, m$, which are tangential to $M$ and their $(1,0)$ component are $\partial / \partial z_{j}$ respectively. $\Delta=\sum_{j=1}^{m} M_{j}^{2}$ is elliptic in a sufficiently small neighborhood of 0 . Consequently, for a given distribution $u$ defined in a neighborhood of 0 , there is a $C^{k}$ function $f$ satisfying $\Delta^{N} f=u$ for a sufficiently large integer $N$. Then, $f=\left.\sum_{j=1}^{l} h_{j}\right|_{M}$, for some holomorphic functions $h_{j}$ defined in some wedges of edge $M$ and continuous up to $M$ from above. Therefore, we have the following holomorphic decomposition $u=\Delta^{N} f=\left.\sum_{j=1}^{l} \Delta_{z}^{N} h_{j}\right|_{M}$, where $\Delta_{z}=\sum_{j=1}^{m} \partial^{2} / \partial z_{j}^{2}$. However, we prefer another way, shown in paragraph 4.3, which makes use of Hörmander's $L^{2}$ estimates of $\bar{\partial}$ solutions.

Remark 2.2.2. As mentioned at the end of (i), the form $G$ is a Tsuno form in the following meaning. Assume that $X$ is the compact support of $f$ in $M$, then, for any neighborhood $\Omega^{\prime} \subset \Omega$ of $X$, and for any holomorphic function $\phi$ in $\Omega$,

$$
\varepsilon \int_{\partial \Omega^{\prime}} G \phi \wedge d z=\int_{X} f \phi d z
$$

where $d z=d z_{1} \wedge \cdots \wedge d z_{m}, \varepsilon= \pm 1$ is independent of $f$. Another way to say this fact is that $f$ is the residue of $\varepsilon G$ on $X$. There are two ways to check the above equality.

(a) "By hand". The right-hand side in the above equality does not depend on the choice of $\Omega^{\prime}$. If we choose $\Omega^{\prime}=\Omega_{n}, n=1,2, \ldots$, so that $\operatorname{dist}\left(X, \partial \Omega_{n}\right) \rightarrow 0$, as $n \rightarrow \infty$, then it follows frrom (2.4) that

$$
\varepsilon \int_{\partial \Omega^{\prime}} G \wedge \phi d z=\lim _{n \rightarrow \infty} \varepsilon \int_{\partial \Omega_{n}} f \phi \chi_{1} \bar{\partial} \chi_{2} \wedge \cdots \wedge \bar{\partial} \chi_{m} \wedge d z
$$

Note that on the intersection (denoted by $S_{n}$ ) of $\partial \Omega_{n}$ and the support of $\chi_{1} \bar{\partial} \chi_{2} \wedge \cdots \wedge \bar{\partial} \chi_{m}$,

$$
\begin{aligned}
f \phi \chi_{1} & \bar{\partial} \chi_{2} \wedge \cdots \wedge \bar{\partial} \chi_{m} \wedge d z=d\left(f \phi \chi_{1} \chi_{2} \wedge \cdots \wedge \bar{\partial} \chi_{m} \wedge d z\right) \\
& -\bar{\partial} f \phi \chi_{1} \chi_{2} \wedge \cdots \wedge \bar{\partial} \chi_{m} \wedge d z-f \phi \chi_{2} \bar{\partial} \chi_{1} \wedge \bar{\partial} \chi_{3} \wedge \cdots \wedge \bar{\partial} \chi_{m} \wedge d z \\
= & d\left(f \phi \chi_{1} \chi_{2} \wedge \cdots \wedge \bar{\partial} \chi_{m} \wedge d z\right)-\bar{\partial} f \phi \chi_{1} \chi_{2} \wedge \cdots \wedge \bar{\partial} \chi_{m} \wedge d z
\end{aligned}
$$


In view of the fact that $\bar{\partial} f$ vanishes on $M$ to $\infty$ order,

$$
\begin{aligned}
\lim _{n \rightarrow \infty} & \int_{\partial \Omega_{n}} f \phi \chi_{1} \bar{\partial} \chi_{2} \wedge \cdots \wedge \bar{\partial} \chi_{m} \wedge d z \\
& =\lim _{n \rightarrow \infty} \int_{\partial S_{n}} f \phi \chi_{1} \chi_{2} \bar{\partial} \chi_{3} \wedge \cdots \wedge \bar{\partial} \chi_{m} \wedge d z \\
& =\lim _{n \rightarrow \infty} \int_{S_{n}^{\prime}} f \phi \bar{\partial} \chi_{3} \wedge \cdots \wedge \bar{\partial} \chi_{m} \wedge d z,
\end{aligned}
$$

where $S_{n}^{\prime}=S_{n} \cap\left\{\chi_{2}=1\right\}$ and $S_{n}$ is defined above. By repeated use of Stokes' formula and the fact that $\bar{\partial} f$ vanishes on $M$, we can prove (2.6).

(b) Indirectly, one can think in the following way. The form $G$ gives a holomorphic cocycle $\left\{G_{j_{1} \cdots j_{m}}\right\}$ (moreover, a holomorphic decomposition of $f$ as in (2.5)). As we will see later (Theorem 4.2.1), any Tsuno form also gives another holomorphic cocycle (a same kind of holomorphic decomposition of $f$ ). Therefore, these two cocycles are cohomologuous in the Čech cohomology; and hence, the corresponding forms are cohomologuous in the Dolbeault cohomology. Thus, the form $G$ itself is a Tsuno form.

\section{BOUNDARY VALUES OF HOLOMORPHIC FUNCTIONS}

3.1. Notations. We try to be a little more ambitious to extend the previous decomposition argument to a more general case, in which we lose any kind of smoothness and hence the usual regularization technique fails. Therefore, we turn to consider a kind of general boundary value of holomorphic functions without growth restrictions, both for its own right and for our later purpose. Following Hörmander [H1], we introduce notions of Martineau's analytic functionals and Sato's hyperfunctions. Denote the set of all functions holomorphic in an open set $U$ of $\mathbf{C}^{m}$ by $A(U)$. Let $X$ be a bounded set in a manifold $M \subset \mathbf{C}^{m}$, then we define the following notions.

Definition 3.1.1. $u$ is called an analytic functional carried by $X$ if $u$ is a linear functional on $A\left(\mathbf{C}^{m}\right)$ and satisfies that for every neighborhood $U_{\delta}=:\{w \in$ $\left.\mathbf{C}^{m}: \operatorname{dist}(w, X)<\delta\right\}$ of $X$ in $\mathbf{C}^{m}$, there is a positive constant $C$ such that

$$
|u(\psi)| \leq C\|\psi\|_{L^{\infty}\left(U_{\delta}\right)}, \quad \forall \psi \in A\left(\mathbf{C}^{m}\right) .
$$

We denote by $A^{\prime}(X)$ the set of all analytic functionals carried by $X$.

Definition 3.1.2. A hyperfunction carried by $X$ is an element in the quotient space $B(X)=: A^{\prime}(X) / A^{\prime}\left(\partial_{M} X\right)$, where $\partial_{M} X$ is understood as the boundary of $X$ in the topology of $M$.

In this section, we consider $M$ to be a totally real $C^{1}$-manifold $\subset \mathbf{C}^{m}$ of real dimension $m, 0 \in M$, which, with suitable coordinates, is given by

$$
M: \zeta \in \mathbf{R}^{m} \mapsto Z(\zeta)=\zeta+i \phi(\zeta) \in \mathbf{C}^{m}
$$

in a neighborhood $U_{0}$ of 0 , where $\phi \in C^{1}\left(\mathbf{R}^{m}\right)$ satisfies

$$
\phi(0)=0, \quad \phi^{\prime}(0)=0 .
$$

Let $\Gamma$ be a cone in $\mathbf{R}^{m}$ with vertex 0 , and $\Gamma_{\rho}=:\{\gamma \in \Gamma:|\gamma|<\rho\}$. Then put

$$
M_{\rho}=\{w \in M:|\operatorname{Re} w|<\rho\}, \quad W_{\rho}(\Gamma)=M_{\rho}+i \Gamma_{\rho} .
$$

As usual, $W_{\rho}(\Gamma)$ is called a wedge of edge $\Gamma$. 
3.2. Boundary values of holomorphic functions without growth restriction. Let $f$ be a holomorphic function defined in $W_{\rho}(\Gamma) \subset U_{0}$, and let $X$ be a compact neighborhood of $0 \Subset M_{\rho}$ in $M$. We now consider the following linear functional:

$$
u_{\gamma}^{X}(\psi)=: \int_{X+i \gamma} f(w) \psi(w) d w, \quad \forall \psi \in A\left(\mathbf{C}^{m}\right),
$$

for $\gamma \in \Gamma_{\rho}$. Then, we obtain

Theorem 3.2.1. There is an analytic functional $u^{X} \in A^{\prime}(X)$, unique up to an element of $A^{\prime}\left(\partial_{M} X\right)$, such that, for any neighborhood $V$ of $\partial_{M} X$ in $\mathbf{C}^{m}$, there is $\delta>0$ satisfying

$$
u^{X}-u_{\gamma}^{X} \in A^{\prime}(V), \quad \forall \gamma \in \Gamma_{\delta} .
$$

The above theorem says, of course, that there is a unique hyperfunction $\overline{u^{X}} \in$ $B(X)$ associated to a function holomorphic in $U+i \Gamma_{\rho}$ for some neighborhood $U$ of $X$ in $M$. We call the hyperfunction $\overline{u^{X}}$ the boundary value of $f$ on the edge $X$, denoted by $B f_{\Gamma}^{X}$. The important reason for calling the hyperfunction $B f_{\Gamma}^{X}$ the boundary value of $f$ is that any analytic functional $u$ in $B f_{\Gamma}^{X}$ can reproduce $f$ in a sufficiently small neighborhood of 0 . If $u \in B f_{\Gamma}^{X}$, we define

$$
u_{\tau}(z)=:\left\langle u,(\tau / 2 \pi)^{m / 2} e^{-\tau[z-\cdot]^{2}}\right\rangle,
$$

where $[z-w]^{2}=\sum_{j=1}^{m}\left(z_{j}-w_{j}\right)^{2}, \tau>0$ (notice that this is the Baouendi-Treves approximation scheme). Then, it follows easily that $u_{\tau} \rightarrow f(z)$, as $\tau \rightarrow \infty$ pointwise for $z$ in a sufficiently small neighborhood of 0 . Therefore, the map $f \mapsto B f_{\Gamma}^{X}$ is one-to-one, and the boundary value determines the holomorphic function conversely.

We remind the reader that the following proof is modified from an argument in [H1]. In his book [H1], Hörmander proved the existence of $B f_{\Gamma}^{X}$ in the case of a straight edge $M$ by relating analytic functionals to their harmonic representations, and using the Runge approximation for harmonic functions and some knowledge of analytic Cauchy problems. To extend Hörmander's argument to non-real-analytic case, we can make use of the Tsuno representations and the Runge approximation for $\bar{\partial}$ closed form. Rather than doing this way, we present here a direct and elementary method, relying only on Taylor's expansion, to prove the existence of the hyperfunction boundary value. As a result, the smoothness of the edge can be minimized (to be even Lipschitz continuous). For convenience, we assume $M$ is $C^{1}$. It is our intention that the reader get an intuitive understanding of hyperfunction boundary values from the proof.

Proof of Theorem 3.2.1. Without loss of generality, we consider a strictly convex $\Gamma_{\rho}$. Since $f, \psi$ are holomorphic in $W_{\rho}(\Gamma)$, it follows from Stokes' formula that for $\gamma, \gamma^{\prime} \in \Gamma_{\rho}$

$$
\left(u_{\gamma}^{X}-u_{\gamma^{\prime}}^{X}\right)(\psi)=\int_{\partial_{M} X+i\left[\gamma, \gamma^{\prime}\right]} f(w) \psi(w) d w,
$$

where $\left[\gamma, \gamma^{\prime}\right]=:\left\{t \gamma+(1-t) \gamma^{\prime}: 0 \leq t \leq 1\right\}$. Let $\gamma \in \Gamma_{\rho}$, and let $\gamma_{j}=$ $\gamma / j, j=1,2, \ldots$ Then, $u_{\gamma_{j+1}}^{X}-u_{\gamma_{j}}^{X}$ is an analytic functional carried by $E_{j}=\partial_{M} X+i[1 /(j+1), 1 / j] \gamma$. The trick here is to construct a sequence of distributions supported on $\partial_{M} X$ to approximate $u_{\gamma_{j+1}}^{X}-u_{\gamma_{j}}^{X}, j=1,2, \ldots$, in 
order to control the growth of $f$ to obtain the analytic functional $u^{X}$. Denote $\psi_{\gamma}(z, \zeta)=\psi(\zeta+i z \gamma)$, where $\zeta \in \partial_{M} X, z \in \mathbf{C}$, then

$$
\psi_{\gamma}(z, \zeta)=\sum_{p=0}^{k} \frac{\partial^{p} \psi_{\gamma}(0, \zeta)}{\partial z^{p}} \frac{z^{p}}{p !}+\frac{1}{k !} \int_{0}^{z}(z-\tau)^{k} \frac{\partial^{k+1} \psi(\tau, \zeta)}{\partial z^{k+1}} d \tau .
$$

Note that

$$
\int_{E_{j}} f(w) \psi(w) d w=\int_{\partial_{M} X} \int_{1 /(j+1)}^{1 / j} f(\zeta+i t \gamma) \psi(\zeta+i t \gamma) \alpha_{j}(\zeta) d t d \sigma(\zeta)
$$

where $\alpha_{j}(\zeta) \in C\left(\partial_{M} X\right), d \sigma$ is an area measure on $\partial_{M} X$. It follows

$$
\begin{array}{rl}
\int_{E_{j}} & f(w) \psi(w) d w=\sum_{p=0}^{k} \int_{\partial_{M} X} \frac{\partial^{p} \psi(0, \zeta)}{\partial z^{p}} \alpha_{j}(\zeta)\left(\int_{1 /(j+1)}^{1 / j} f(\zeta+i t \gamma) \frac{t^{p}}{p !} d t\right) d \sigma(\zeta) \\
& +\int_{\partial_{M} X} \int_{1 /(j+1)}^{1 / j} \alpha_{j}(\zeta) f(\zeta+i t \gamma) \int_{0}^{t} \frac{\partial^{k+1} \psi_{\gamma}(\tau, \zeta)}{\partial z^{k+1}} \frac{(t-\tau)^{k}}{k !} d \tau d t d \sigma(\zeta) \\
& =\sum_{p=0}^{k} \int_{\partial_{M} X} a_{p, j}(\zeta) \frac{\partial^{p} \psi_{\gamma}(0, \zeta)}{\partial z^{p}} d \sigma(\zeta)+R_{k, j}(\psi)
\end{array}
$$

where $a_{p, j}(\zeta) \in C\left(\partial_{M} X\right)$. By Cauchy's estimate, for $1 /(j+1) \leq \tau \leq 1 / j$,

$$
\left|\frac{\partial^{k+1} \psi_{\gamma}(\tau, \zeta)}{\partial z^{k+1}}\right| \leq \frac{k !}{(2 / j)^{k+1}} \max _{|z| \leq 3 / j}\left|\psi_{\gamma}(z, \zeta)\right|
$$

and consequently, there is a positive constant $C_{j}$ such that

$$
\left|R_{k, j}(\psi)\right| \leq\left(C_{j} / 2^{k}\right)\|\psi\|_{L^{\infty}\left(V_{j}\right)}, \quad \forall \psi \in A\left(\mathbf{C}^{m}\right), k=1,2, \ldots ;
$$

where $V_{j}=\left\{w \in \mathbf{C}^{m}: \operatorname{dist}\left(w, \partial_{M} X\right)<\frac{3}{j}|\gamma|\right\}, j=1,2, \ldots$ Notice that

$$
\psi \mapsto \sum_{p=0}^{k} \int_{\partial_{M} X} a_{p, j}(\zeta) \frac{\partial^{p} \psi_{\gamma}(0, \zeta)}{\partial z^{p}} d \sigma(\zeta)
$$

is a distribution supported on $\partial_{M} X$. Therefore, for each $j$, we can find a distribution $v_{j}$ supported on $\partial_{M} X$ such that

$$
\left|\left(u_{\gamma_{j+1}}^{X}-u_{\gamma_{j}}^{X}-v_{j}\right)(\psi)\right| \leq\left(1 / 2^{j}\right)\|\psi\|_{L^{\infty}\left(V_{j}\right)}, \quad \forall \psi \in A\left(\mathbf{C}^{m}\right) .
$$

Next, construct the following sequence of analytic functionals:

$$
w_{n}=u_{\gamma_{1}}^{X}+\sum_{j=1}^{n}\left(u_{\gamma_{j+1}}^{X}-u_{\gamma_{j}}^{X}-v_{j}\right), \quad n=2,3, \ldots
$$

(Note that this is the Mittag-Leffler procedure.) Then, for every $\psi \in A\left(\mathbf{C}^{m}\right)$, $\left\{w_{n}(\psi)\right\}_{n \geq 2}$ forms a Cauchy sequence; and so, there is a linear functional $u^{X}$ defined on $A\left(\mathbf{C}^{m}\right)$ such that

$$
\lim _{n \rightarrow \infty} w_{n}(\psi)=u^{X}(\psi), \quad \forall \psi \in A\left(\mathbf{C}^{m}\right) .
$$

Notice that, for any $n \in \mathbf{N}$,

$$
u^{X}(\psi)=u_{\gamma_{n}}^{X}(\psi)-v_{1}(\psi)-\cdots-v_{n-1}(\psi)+\sum_{j=n}^{\infty}\left(u_{\gamma_{j+1}}^{X}-u_{\gamma_{j}}^{X}-v_{j}\right)(\psi)
$$


We can conclude that a carrier of $u^{X}$ is $X$ and $u^{X}-u_{\gamma_{n}}^{X}$ is an analytic functional carried by the neighborhood $V_{n}$ of $\partial_{M} X$. From (3.5), we can see that, for any neighborhood $V$ of $\partial_{M} X$, there is a $\delta_{V}>0$ such that $u^{X}-u_{\gamma}^{X} \in A^{\prime}(V)$, $\forall \gamma \in \Gamma_{\delta_{V}}$ as desired. Uniqueness of $u^{X}$ is trivial.

Definition 3.2.1. Let $u \in A^{\prime}(X)$, and let $X_{1} \subset X$ be nonempty, then $v \in A^{\prime}\left(X_{1}\right)$ $\left(\subset A^{\prime}(X)\right)$ is called a restriction of $u$ to $X_{1}$ if $u-v \in A^{\prime}\left(X \backslash X_{1}\right)$.

Remark 3.2.1. The existence of restrictions for general analytic functionals will be treated in $\S 6$, which is independent of the rest of the paper. We do not speak about the restriction of $u$ to avoid discussing the question of uniqueness for the time being. In fact, unless we shrink $X$ enough, uniqueness may fail (due to the fact that a totally real manifold is only locally polynomially convex). In our local setting, the set $X$ is polynomially convex, and so is its subset $X_{1}$. According to the discussion in the proof of Corollary 6.3.1, there is a restriction of $u \in A^{\prime}(X)$ to $X_{1}$, which is also unique up to an element in $A^{\prime}\left(\partial X_{1}\right)$. Therefore, there is a unique element in $B(X)$ consisting of all restrictions of $u$ to $X_{1}$, which will be denoted by $\left.u\right|_{X_{1}}$.

We consider the following interesting case which shows us the relation between $B f_{\Gamma}^{X_{1}}$ and $B f_{\Gamma}^{X}$ for $X_{1} \subset X$. It is easy to prove the following proposition from the above theorem.

Proposition 3.2.1. Given two compact (open) sets $X_{1} \subset X \subset M_{\rho}$, if $f$ is holomorphic in $W_{\rho}(\Gamma)$, then any analytic functional in $B f_{\Gamma}^{X_{1}}$ is a restriction of analytic functionals in $B f_{\Gamma}^{X}$ to $X_{1}$.

Proof. For any neighborhood $V$ of $\overline{X \backslash X_{1}}$, choose $\gamma \in \Gamma_{\rho}$ with $|\gamma|$ small enough that for any $u^{X} \in B f_{\Gamma}^{X}$ and any $u^{X_{1}} \in B f_{\Gamma}^{X_{1}}$,

$$
u_{\gamma}^{X}-u^{X} \in A^{\prime}(V), \quad u_{\gamma}^{X_{1}}-u^{X_{1}} \in A^{\prime}(V), \quad u_{\gamma}^{X_{1}}-u_{\gamma}^{X} \in A^{\prime}(V),
$$

since

$$
\left(u_{\gamma}^{X_{1}}-u_{\gamma}^{X}\right)(\psi)=\int_{X \backslash X_{1}+i \gamma} f(w) \psi(w) d w
$$

Therefore,

$$
u^{X}-u^{X_{1}}=\left(u^{X}-u_{\gamma}^{X}\right)+\left(u_{\gamma}^{X}-u_{\gamma}^{X_{1}}\right)+\left(u_{\gamma}^{X_{1}}-u^{X_{1}}\right) \in A^{\prime}(V),
$$

which shows the proposition.

As an example of the hyperfunction boundary value, we note that when $f$ is continuous up to the edge $M$, then $\left.\chi_{X} f\right|_{M}$ is a representative in $B f_{\Gamma}^{X}$, where we denote by $\chi_{X}$ the characteristic function of the set $X$.

\section{DeCOMPOSITION IN MORE GENERAL SENSE}

4.1. Tsuno's representation formula. Tsuno's representation formula will play a major role in the paper. For distributions with compact support, it is actually an absolutely immediate consequence of the Bochner-Martinelli representation formula for holomorphic functions. As before, let $X$ be a compact set in $M$, a $C^{\infty}$ smooth totally real manifold, and $u$ a distribution with compact support in $X$ (or $M$ is $C^{1}$ and $u$ a function with compact support). Then, for any 
neighborhood $U$ of $X$ in $\mathbf{C}^{m}$, for any holomorphic function $\psi$, we have

$$
\begin{aligned}
\langle u, \psi\rangle & =\left\langle u, \int_{\partial U} B M(\cdot, w) \psi(w) \wedge d w\right\rangle \\
& =\int_{\partial U}\langle u, B M(\cdot, w)\rangle \psi(w) \wedge d w
\end{aligned}
$$

where

$$
B M(z, w)=\frac{1}{(2 \pi i)^{m}} \frac{\sum_{j=1}^{m}(-1)^{j+1}\left(\bar{w}_{j}-\bar{z}_{j}\right) d \bar{w}_{1} \wedge \cdots \wedge \widehat{d \bar{w}}_{j} \wedge \cdots \wedge d \bar{w}_{m}}{|w-z|^{2 m}}
$$

is the Bochner-Martinelli kernel, and the pairing $\langle u, \psi\rangle$ is so defined that, for any function $u$,

$$
\langle u, \psi\rangle=\int_{X} u \psi d z .
$$

Then, the kernel $G(w)=\langle u, B M(\cdot, w)\rangle$ is a representation of the distribution $u$, which is a $\bar{\partial}$ closed $(0, m-1)$ form in $\mathbf{C}^{m} \backslash X$. It needs some work to extend this representation formula to the analytic functional case since the BochnerMartinelli kernel is not holomorphic unless in $\mathbf{C}^{1}$. This has been done by Tsuno [Ts], who also gives credits to Harvey and Martineau, using Henkin's integral representation formula and technique of solving Cousin I problem.

Theorem 4.1.1 [Ts]. Let $X$ be a compact polynomially convex set, and let $u \in$ $A^{\prime}(X)$, then there is a $\bar{\partial}$ closed $(0, m-1)$ form $G$ defined in $\mathbf{C}^{m} \backslash X$, so that, for every bounded neighborhood $U$ of $X$ in $\mathbf{C}^{m}$, and for every holomorphic function $\psi$,

$$
\langle u, \psi\rangle=\int_{\partial U} G(w) \psi(w) \wedge d w .
$$

Moreover, any two such representation forms differ by a $\bar{\partial}$ exact form defined in $\mathbf{C}^{m} \backslash X$.

Definition 4.1.1. A $\bar{\partial}$ closed $(0, m-1)$ form $G$ defined in $\mathbf{C}^{m} \backslash X$ is called a Tsuno representation form of $u \in A^{\prime}(X)$ in this paper if $G$ satisfies the relation (4.1) for any holomorphic function $\psi$.

In case that $X=K_{1} \times K_{2} \times \cdots \times K_{m}$ for some polynomially convex compact sets $K_{j}, j=1, \ldots, m$, the above theorem is proved by Harvey [Hv2], and the form $G$ can be given as the Cauchy transform of $f$ in multidimension setting. The following proof of the above theorem is essentially taken from [Ts] for convenience of reference.

Proof of Theorem 4.1.1. The uniqueness is straightforward from the following lemma (it will also be used to prove the existence).

Lemma 4.1.1. Let $\Omega$ be polynomially convex and have a strictly pseudoconvex neighborhood basis. Then, a $\bar{\partial}$ closed $(0, m-1)$ form $G$ in $\mathbf{C}^{m} \backslash \Omega$ is $\bar{\partial}$ exact if and only if, for any neighborhood $U$ of $\Omega$ and any holomorphic function $\psi$,

$$
\int_{\partial U} G \wedge \psi d w=0
$$

The proof of the lemma will be given after the proof of the theorem. Let us now prove the existence of Tsuno forms. 
(i) Let $\left\{U_{j}\right\}_{j=1}^{\infty}$ be a strictly pseudoconvex neighborhood basis of $X$ in $\mathbf{C}^{m}$ such that $U_{j+1} \Subset U_{j}, j=1,2, \ldots, \bigcap_{j=1}^{\infty} U_{j}=X$. For each $j$, there is a Henkin-Ramirez kernel $H_{j}(z, w)$ for the domain $U_{j+1}$, which is holomorphic in $z$ for $z \in U_{j+1}, \bar{\partial}$ closed in $w$ for $w \in U_{j} \backslash U_{j+1}$, and satisfies that, for every holomorphic function $\psi$,

$$
\psi(z)=\int_{\partial U_{j+1}} H_{j}(z, w) \psi(w) \wedge d w .
$$

Set

$$
R_{j}(w)=\left\langle u, H_{j}(\cdot, w)\right\rangle .
$$

Then, $R_{j}$ is a $\bar{\partial}$ closed $(0, m-1)$ form in $U_{j} \backslash U_{j+1}$ and satisfies

$$
\langle u, \psi\rangle=\int_{\partial U_{j+1}} R_{j}(z, w) \psi(w) \wedge d w .
$$

(ii) The form $R_{j}$ can be extended to $\mathbf{C}^{m} \backslash U_{j+1}$ in the following way by solving a Cousin I problem. Choose a cut-off function $\chi \in C_{0}^{\infty}\left(U_{j}\right)$ which is identically 1 in a neighborhood of $U_{j+1}$ in $\mathbf{C}^{m}$. Then, $\bar{\partial} \chi \wedge R_{j}$ is a $(0, m)$ form defined globally in $\mathbf{C}^{m}$. We can find subsequently a $(0, m-1)$ form $g$ defined in $\mathbf{C}^{m}$ so that $\bar{\partial} g=\bar{\partial} \chi \wedge R_{j}$. Let $G_{1}=\chi R_{j}-g, G_{2}=(1-\chi) R_{j}+g$. Then, $G_{1}$ is $\bar{\partial}$ closed in $\mathbf{C}^{m} \backslash U_{j+1}, G_{2}$ is $\bar{\partial}$ exact in $U_{j}$ since $U_{j}$ is pseudoconvex, and $R_{j}=G_{1}+G_{2}$. Therefore, $G_{1}$ serves as an extension of $R_{j}$, i.e., (4.3) still holds with $G_{1}$ in place of $R_{j} . G_{1}$ will be written as $\omega_{j}$.

(iii) Since

$$
\int_{\partial U} \omega_{j} \psi=\int_{\partial U} \omega_{j+1} \psi=\langle u, \psi\rangle
$$

for any holomorphic function $\psi$ and any neighborhood $U$ of $U_{j}$, we see that $\omega_{j}-\omega_{j+1}$ satisfies the hypothesis of the above lemma with $U_{j}$ in place of $\Omega$ provided $j$ is sufficiently large. Then, there is a $(0, m-2)$ form $\gamma_{j}$ on $\mathbf{C}^{m}$ so that $\omega_{j}=\omega_{j+1}+\bar{\partial} \gamma_{j}$, hence, $\omega_{j}$ can be extended to $U_{j+1}^{c}$ and therefore successively to $\mathbf{C}^{m} \backslash X$. Thus, a Tsuno form is obtained.

Proof of lemma. Necessity is trivial. As is well known, the condition in the lemma is exactly the Greiner-Kohn-Stein condition for $\bar{\partial}_{b}$ solvability. The proof of the sufficiency is a Serre duality argument taken essentially from a personal communication of $\mathrm{H}$. Alexander to Rosay and Stout. Let $\left\{\boldsymbol{\Omega}_{j}\right\}_{j=1}^{\infty}$ be a strictly pseudoconvex neighborhood basis of $\Omega, \Omega_{j+1} \Subset \Omega_{j}$. Choose a $C^{\infty}$ function $\chi_{j}$ so that $\left.\chi_{j}\right|_{\Omega_{j+1}}=0,\left.\chi_{j}\right|_{\Omega_{j}^{c}}=1$. Then, $\chi_{j} G$ is well defined on $\mathbf{C}^{m}$. $\bar{\partial}\left(\chi_{j} G\right)=\bar{\partial} \chi_{j} \wedge G$ is identically 0 in $\Omega_{j}^{c}$ and $\Omega_{j+1}$. Moreover, $\bar{\partial}\left(\chi_{j} G\right)$ is orthogonal to the space of holomorphic functions in $\Omega_{j-1}$ since

$$
\int_{\Omega_{j-1}} \bar{\partial}\left(\chi_{j} G\right) \psi=\int_{\Omega_{j} \backslash \Omega_{j+1}} \bar{\partial}(\chi \psi G)=\int_{\partial \Omega_{j}} \psi G=0
$$

for any holomorphic function $\psi$ in $\Omega_{j-1}$ by the hypothesis of the lemma. Then, Serre's duality theorem $\left(H_{*}^{0, m}\left(\Omega_{j-1}\right) \cong\left[H^{0,0}\left(\Omega_{j-1}\right)\right]^{*}\right)$ [Ser] yields that there is a $(0, m-1)$ form $\alpha_{j}$ with compact support in $\Omega_{j-1}$ so that $\bar{\partial} \alpha_{j}=$ $\bar{\partial}\left(\chi_{j} G\right)$. Note that $\chi_{j} G-\alpha_{j}$ is defined and $\bar{\partial}$ closed on $\mathbf{C}^{m}$ once $\alpha_{j}$ is extended by 0 . It follows that there is a $(0, m-2)$ form $\beta_{j}$ defined on $\mathbf{C}^{m}$ 
so that $\bar{\partial} \beta=\chi_{j} G-\alpha_{j}$. Therefore, $G=\bar{\partial} \beta_{j}$ on $\Omega_{j-1}^{c}$. By induction on $j$, we can conclude $G$ is $\bar{\partial}$ exact on $\mathbf{C}^{m} \backslash \Omega$. The reason is the following. $\beta_{j+1}-\beta_{j}$ is a $\bar{\partial}$ closed $(0, m-2)$ form on $\Omega_{j-1}^{c}$. This is again $\bar{\partial}$ exact on $\Omega_{j-1}^{c}$ basically by the same Serre duality argument as above except for using $H_{*}^{0, m-1}\left(\Omega_{j}\right) \cong\left[H^{0,1}\left(\Omega_{j}\right)\right]^{*}$. Therefore, $\beta_{j}$ can be extended step by step up to $\mathbf{C}^{m} \backslash X$.

We will assume that $X$ is a very small compact neighborhood of 0 in the manifold $M$ so that every closed subset of $X$ is polynomially convex from now on. In this case, $X$ has a strictly pseudoconvex neighborhood basis (see, for example, [HL, Theorem 1.4.18]). Therefore, the above theorem applies.

Remark 4.1.1. It is not essential that $G$ is defined on the whole $\mathbf{C}^{m} \backslash X$. In the paper, we often use a locally defined form $G$, i.e., there is a pseudoconvex neighborhood $\Omega$ of $X$ in $\mathbf{C}^{m}$ such that $G$ is defined in $\Omega \backslash X$ and the relation (4.1) holds for any neighborhood $U$ contained in $\Omega$. Such locally defined form will still be called a Tsuno representation form of $u$ due to the fact that any such locally defined form can be extended to be a globally defined $\bar{\partial}$ closed form, i.e., a form defined in $\mathbf{C}^{m} \backslash X$, modulo a $\bar{\partial}$ exact form defined in $\Omega$, since a $\bar{\partial}$ exact form contributes nothing to the duality formula (4.1) essentially. The way to do such an extension is to solve a simple Cousin problem in $\Omega \backslash X$ as shown in (ii) of the above proof.

Remark 4.1.2. We know at least three ways of constructing Tsuno forms:

(a) the first one is by Bochner-Martinelli kernels as described above;

(b) the second is by hand following Amar's technique (see $\S 2.2$ );

(c) the third is using a given holomorphic decomposition of an analytic functional (see Remark 4.2.3 or the proof of Theorem 5.2.1).

Remark 4.1.3. Let us note the relation between the Tsuno forms of two different representatives for the same element in $B(X)$. Suppose that $u_{1}, u_{2} \in A^{\prime}(X)$ are equivalent, i.e. $u_{1}-u_{2} \in A^{\prime}(\partial X)$, and $G_{1}, G_{2}$ are Tsuno forms for $u_{1}, u_{2}$ respectively. Then, according to Tsuno's theorem, $G_{1}-G_{2}$ is $\bar{\partial}$ closed in $\mathbf{C}^{m} \backslash \partial X$ since it is a Tsuno form of $u_{1}-u_{2}$.

Remark 4.1.4. If two $\bar{\partial}$ closed $(0, m-1)$ form $G_{1}, G_{2}$ in $\mathbf{C}^{m} \backslash X$ coincide in the cylinder $\Omega \backslash X$, where $\Omega=X+i D_{m}, D_{m}$ is the closed unit $m$-ball in $\mathbf{R}^{m}$, then it follows immediately from the duality formula (4.1) that $G_{1}, G_{2}$ represents analytic functionals in the same hyperfunction class in $B(X)$. This is actually an indication of a more general fact: any $\bar{\partial}$ closed $(0, m-1)$ form $g$ in $\Omega \backslash X$ represents a hyperfunction class $\bar{u} \in B(X)$. If we define an analytic functional $u_{\delta}$ by setting $\left\langle u_{\delta}, \psi\right\rangle=\int_{S_{\delta}} g \wedge \psi d w$, where $S_{\delta}=X+$ $i\left\{x \in \mathbf{R}^{m}|| x \mid=\delta\right\}$ and $\delta>0$ is a small number, then there exists a hyperfunction class $\bar{u} \in B(X)$ so that $u \in \bar{u}$ if and only if

$$
u-u_{\delta} \text { is carried by } S_{\delta}^{\prime}=\partial X+i \delta D_{m} .
$$

This fact can be proved in the same way as Theorem 3.2.1. Therefore, the restriction of a Tsuno form to the cylinder $\Omega \backslash X$ is very essential in the hyperfunction sense.

4.2. Holomorphic decompositions in general sense. We attempt to develop a holomorphic decomposition theorem for analytic functionals supported on a 
$C^{2}$ totally real manifold $M$. As we have already seen in $\S 3.2$, the analytic functionals arise naturally as the boundary values of holomorphic functions. Now, let $u \in A^{\prime}(X)$ with $X$ a compact neighborhood of 0 in $M$, then there is a $\bar{\partial}$ closed $(0, m-1)$ form $G$ representing $u$ in the fashion (4.1). As in $\S 2, G$ results in a holomorphic cocycle $\left\{G_{j_{1} \cdots j_{m}}, j_{q}= \pm q\right\} \in C^{m-1}(\mathscr{U}, \mathscr{O})$. We then assert that $u$ is a sum of the boundary values of $G_{j_{1} \cdots j_{m}}$ on the edge $M$ in the hyperfunction sense. The proof of the following theorem just makes use of the Stokes' formula repeatedly although we need to be careful about the orientations of the manifolds involved in the proof.

Theorem 4.2.1. With $u$ and $G_{i_{1} \cdots i_{m}}$ as above, we have

$$
u \in \varepsilon \sum_{\substack{i_{p}= \pm p \\ p=1, \ldots, m}} \operatorname{sgn}\left(i_{1} \cdots i_{m}\right)\left(B G_{i_{1} \cdots i_{m}}\right)_{U_{i_{1}} \cdots i_{m}}^{X}
$$

where $\varepsilon= \pm 1$ is independent of $u$.

Proof. Let $I_{\delta}^{m}=[-\delta, \delta]^{m}, \delta>0$, a small number, and let

$$
X_{\delta}=\{z \in M \mid \operatorname{dist}(z, X)<\delta\}
$$

then

$$
X_{\delta} \times I_{\delta}^{m}=:\left\{z+i t \mid z \in X_{\delta}, t \in I_{\delta}^{m}\right\}
$$

is a neighborhood of $X$ in $\mathbf{C}^{m}$. Denote

$$
\begin{aligned}
S_{j} & =\left\{t=\left(t_{1}, \ldots, t_{m}\right) \in I_{\delta}^{m} \mid t_{j}=\delta\right\}, \\
S_{-j} & =\left\{t=\left(t_{1}, \ldots, t_{m}\right) \in I_{\delta}^{m} \mid t_{j}=-\delta\right\}, \quad j=1, \ldots, m .
\end{aligned}
$$

Then, $X_{\delta} \times S_{j}$ is a face of the curved parallelepipeds $X_{\delta} \times I_{\delta}^{m}$. Without loss of generality, we can assume that for all $\delta$ small, $X_{\delta} \times S_{j} \subset U_{j}, j= \pm 1, \ldots, \pm m$. Let $G$ be a representation $(0, m-1)$ form of $u$, then, for all holomorphic functions $\psi$,

$$
u(\psi)=\int_{\partial\left(X_{\delta} \times I_{\delta}^{m}\right)} G(\zeta) \psi(\zeta) \wedge d \zeta .
$$

If $S_{j}$ is given the orientation induced as the boundary of $I_{\delta}^{m}$, we have

$$
u(\psi)=\varepsilon_{1} \sum_{j= \pm 1, \ldots, \pm m} \int_{X_{\delta} \times S_{j}} G_{j}(\zeta) \wedge \psi(\zeta) d \zeta+\int_{\partial X_{\delta} \times I_{\delta}^{m}} G(\zeta) \wedge \psi(\zeta) d \zeta
$$

where $\varepsilon_{1}= \pm 1$ arises from the orientation of the product manifold.

Note that $\psi \mapsto \int_{\partial X_{\delta} \times I_{\delta}^{m}} G(\zeta) \wedge \psi(\zeta) d \zeta$ defines a distribution supported on $\partial X_{\delta} \times I_{\delta}^{m}$ which is in a small neighborhood of $\partial_{M} X$. As we have seen in the proof of Theorem 3.2.1, this analytic functional converges to 0 in the hyperfunction sense as $\delta \rightarrow 0$. Therefore, we only need to take care of the main term

$$
A=: \sum_{j= \pm 1, \ldots, \pm m} \int_{X_{\delta} \times S_{j}} G_{j}(\zeta) \wedge \psi(\zeta) d \zeta .
$$

We will show that

$$
A \equiv \varepsilon \sum_{\substack{i_{p}= \pm p \\ p=1, \ldots, m}} \int_{X_{\delta} \times S_{i_{1} \cdots i_{m}}} G_{i_{1} \cdots i_{m}}(\zeta) \wedge \psi(\zeta) d \zeta \quad \bmod \left(A^{\prime}\left(\partial X_{\delta} \times I_{\delta}^{m}\right)\right)
$$


where $S_{i_{1} \cdots i_{p}}=: S_{i_{1}} \cap \cdots \cap S_{i_{p}}$ carries the orientation induced as the boundary of $S_{i_{2} \cdots i_{p}}$ inductively.

Suppose (4.6) is proved, one easily sees that $X_{\delta} \times S_{i_{1}} \cdots i_{m}$ is parallel to $X_{\delta}$ and its orientation is $\operatorname{sgn}\left(i_{1} \cdots i_{m}\right) \cdot\left\{\right.$ the orientation of $\left.X_{\delta} \times S_{1 \cdots m}\right\}$. Let $\delta \rightarrow 0$, the conclusion of the theorem follows easily from the definition of the hyperfunction boundary value.

Next, we prove (4.6).

From the standard orientation consideration of a simplex, it follows that

$$
S_{i_{1} \cdots i_{k}}=\varepsilon_{j_{1} \cdots j_{k}}^{i_{1} \cdots i_{k}} S_{j_{1} \cdots j_{k}}
$$

where

$$
\varepsilon_{j_{1} \cdots j_{k}}^{i_{1} \cdots i_{k}}=: \begin{cases}0 & \text { if }\left\{i_{1} \cdots i_{k}\right\} \neq\left\{j_{1} \cdots j_{k}\right\}, \\
\operatorname{sgn}\left(\begin{array}{l}
i_{1} \cdots i_{k} \\
j_{1} \cdots j_{k}
\end{array}\right) \text { if }\left\{i_{1} \cdots i_{k}\right\}=\left\{j_{1} \cdots j_{k}\right\} .\end{cases}
$$

Carrying the Weil process described in 2.1 onto the integrals in $A$, we have

$$
\begin{aligned}
A & =\sum_{j= \pm 1, \ldots, \pm m} \int_{X_{\delta} \times S_{j}} \bar{\partial} F_{j}(\zeta) \wedge \psi d \zeta \\
& \equiv \varepsilon_{1} \sum_{\left|j_{1}\right|<\left|j_{2}\right|} \int_{X_{\delta} \times S_{j_{1} j_{2}}}\left(F_{j_{2}}-F_{j_{1}}\right)(\zeta) \wedge \psi(\zeta) d \zeta \bmod \left(A^{\prime}\left(\partial X_{\delta} \times I_{\delta}^{m}\right)\right) \\
& \vdots \\
& \equiv \varepsilon_{p-1} \sum_{\left|j_{1}\right|<\cdots<\left|j_{p}\right|} \int_{X_{\delta} \times S_{j_{1} \ldots j_{p}}} \bar{\partial} F_{j_{1} \cdots j_{p}}(\zeta) \wedge \psi(\zeta) d \zeta \bmod \left(A^{\prime}\left(\partial X_{\delta} \times I_{\delta}^{m}\right)\right) \\
& \equiv \varepsilon_{p} \sum_{\left|j_{1}\right|<\cdots<\left|j_{p+1}\right|} \int_{X_{\delta} \times S_{j_{1} \cdots j_{p+1}}} G_{j_{1} \cdots j_{p+1}}(\zeta) \wedge \psi(\zeta) d \zeta \bmod \left(A^{\prime}\left(\partial X_{\delta} \times I_{\delta}^{m}\right)\right)
\end{aligned}
$$

To see the final equivalence above, notice that only $F_{j_{1} \cdots \hat{j}_{k} \cdots j_{p+1}}$ contributes to the integral over $X_{\delta} \times S_{j_{1} \cdots j_{p+1}}$, and more precisely,

$$
\begin{aligned}
\varepsilon_{p-1} & \int_{X_{\delta} \times S_{j_{1}} \cdots j_{k} \cdots j_{p+1}} \bar{\partial} F_{j_{1} \cdots \hat{j}_{k} \cdots j_{p+1}} \wedge \psi d \zeta \\
\equiv & \varepsilon_{p} \int_{X_{\delta} \times \partial S_{j_{1} \cdots j_{k} \cdots j_{p+1}}} F_{j_{1} \cdots \hat{j}_{k} \cdots j_{p+1}} \wedge \psi d \zeta \quad \bmod \left(A^{\prime}\left(\partial X_{\delta} \times I_{\delta}^{m}\right)\right) \\
\equiv & \varepsilon_{p} \int_{X_{\delta} \times S_{j_{k}, j_{1} \cdots j_{k} \cdots j_{p+1}}} F_{j_{1} \cdots \hat{j}_{k} \cdots j_{p+1}} \wedge \psi d \zeta \\
& +\left\{\text { the other integrals over } \partial S_{j_{1} \cdots \hat{j}_{k} \cdots j_{p+1}}\right\} \bmod \left(A^{\prime}\left(\partial X_{\delta} \times I_{\delta}^{m}\right)\right) \\
= & \varepsilon_{p} \int_{X_{\delta} \times S_{j_{1} \cdots j_{p+1}}}(-1)^{k+1} F_{j_{1} \cdots j_{k} \cdots j_{p+1}} \wedge \psi d \zeta \\
& +\left\{\text { integrals not over } X_{\delta} \times S_{j_{1} \cdots j_{p+1}}\right\},
\end{aligned}
$$

finally, adding all these first terms, we obtain the above recurrence relation. Therefore, we end with

$$
A \equiv \varepsilon_{m-1} \sum_{\left|j_{1}\right|<\cdots<\left|j_{m}\right|} \int_{X_{\delta} \times S_{j_{1}} \cdots j_{m}} G_{j_{1} \ldots j_{m}}(\zeta) \wedge \psi(\zeta) d \zeta \quad \bmod \left(A^{\prime}\left(\partial X_{\delta} \times I_{\delta}^{m}\right)\right),
$$

which is $(4.6)$. 
Remark 4.2.1. The above theorem is clearly a kind of generalization of Martineau's decomposition theorem of analytic functionals (see, for example, $[\mathrm{H} 1$, Theorem 9.3.7]). As a corollary of the theorem, we have that the hyperfunction space $B(X)$ is locally spanned by the boundary values of holomorphic functions in certain wedges of edge $X$. In the case $M=\mathbf{R}^{m}$, this fact is taken as the definition of Sato's hyperfunctions. Therefore, the hyperfunction defined here is a natural extension of Sato's object. We also mention the work of Harvey and Wells [Hv1, HvWe]. In $\S 6$, we shall discuss the flabbiness of the sheaf of the germs of hyperfunctions defined here by using the explicit Tsuno representation formula, which should be considered as an elementary presentation of the sheaf cohomology approach employed in [Hvl, HvWe].

Remark 4.2.2. The above theorem (and its distribution case in the next paragraph) can be strengthened as follows. Given any Stein covering $\left\{U_{j}\right\}_{j=1}^{l}$ of $\Omega \backslash M$, we can decompose $u$ as a sum of the boundary values of holomorphic functions defined in $U_{i_{1} \cdots i_{m}}, 1 \leq i_{1}<i_{2}<\cdots<i_{m} \leq l$. Notice also that $U_{i_{1} \cdots i_{m}}$ plays the role of convex wedges in the straight edge case. For this reason and for convenience, $U_{i_{1} \cdots i_{m}}$ will be called a pseudo-wedge of edge $M$. As a matter of fact, such kind pseudo-wedge can be exhausted by the usual wedges after possible shrinking of neighborhoods of 0 , and vice versa due to the fact that $\rho_{j}$ can be replaced by any of the convex combinations of $\rho_{i}$ 's.

Remark 4.2.3. When starting with a holomorphic cocycle $\left\{h_{j_{1} \cdots j_{m}}\right\}$, we can obtain a $\bar{\partial}$ closed $(0, m-1)$ form $G_{1}$ in $\mathbf{C}^{m} \backslash X$ following the Weil process (or simply solving Cousin I problems). Then, the argument in the proof provides us a way to calculate the residue of $G_{1}$, which is a sum of the boundary values of $h_{j_{1} \cdots j_{m}}$ modulo $A^{\prime}\left(\partial_{M} X\right)$. Therefore, if $\left\{h_{j_{1} \cdots j_{m}}\right\}$ is a holomorphic decomposition of an analytic functional $u$ (its Tsuno representation is $G$ ), then the resulted form $G_{1}$ differs from $G$ by a $\bar{\partial}$ closed form in $\mathbf{C}^{m} \backslash \partial_{M} X$. Thus, if restricting ourselves in a smaller neighborhood $X_{1}(\subset X)$ of $0, G_{1}$ and $G$ are cohomologuous. Moreover, $G_{1}$ can be taken as a Tsuno representation of $u$.

\section{5. (HYPO) ANALYTIC WAVE FRONT SET}

5.1. Definition. Since an analytic functional is related to its Tsuno representation form by a duality formula, in principle, any information on the analytic functional should be reflected by its Tsuno form. In this section, we will tell how the wave front set is determined by the representation form. To define the wave front sets of hyperfunctions in case $M$ is $C^{2}$ smooth, we follow Sato's approach.

Definition 5.1.1. Let $u \in A^{\prime}(X)$ with $X$ a compact neighborhood of 0 in $M_{\rho}$. We say that $\sigma \in \mathbf{R}^{m} \backslash\{0\}$ is not in the (analytic) wave front set of $u$ at 0 , denoted by $\sigma \notin W F_{0}(u)$, if there are $r>0$, cones $\Gamma_{j} \subset \mathbf{R}^{m}$ with vertex 0 , and holomorphic functions $h_{j} \in A\left(W_{r}\left(\Gamma_{j}\right)\right), j=1, \ldots, l$, such that

(i) $M_{r} \subset X$,

(ii) $\left.u\right|_{M_{r}}=\sum_{j=1}^{l}\left(B h_{j}\right)_{\Gamma_{j}}^{M_{r}}$,

(iii) for each $j$, there is $\gamma_{j} \in \Gamma_{j}$ so that $\sigma \cdot \gamma_{j}<0, j=1, \ldots, l$.

It is easy to see that one can replace the wedges $W_{r}\left(\Gamma_{j}\right)$ by corresponding 
pseudo-wedges as defined in Remark 4.2.2. We thus obtain an equivalent definition in terms of pseudo-wedges. In this sense, we will not make a difference between pseudo-wedges and wedges in the context whenever no confusion happens.

In [BRT], wave front set is defined in the distribution language. Due to an equivalent definition in terms of FBI transforms, the notions of the wave front sets of a distribution in the above sense and in the distribution sense turn out to be the same.

The main purpose of this section is to present another characterization of the wave front set in terms of $\bar{\partial}$ exactness of the Tsuno representation forms, and to provide its application to holomorphic extension problem from the totally real manifold $M$. One could find that the way adopted here is closely tied with the monodromy phenomenon in $\mathbf{C}^{1}$. As a consequence, we give a hyperfunction version of the edge-of-the-wedge theorem with a $C^{2}$ totally real edge which extends Pincuk's results for distributions (see [P] or Appendix).

5.2. $\bar{\partial}$ exactness for Tsuno representation forms and wave front. Let us first exploit the relation between the wave front set of an analytic functional $u \in$ $A^{\prime}(X)$ and its Tsuno representation form $G$ in (4.1).

Theorem 5.2.1. Let $u \in A^{\prime}(X)$, and let $G$ be a Tsuno representation $(0, m-1)$ form of $u$, then $\sigma_{0} \notin W F_{0}(u)$ if and only if there is a concave cone $\Gamma$ containing $\sigma_{0}$ so that $\sigma_{0} \cdot \partial \Gamma<0$, and there is a smooth $(0, m-2)$ form $g$ in $W_{\rho}(\Gamma)$ for some $p>0$ with

$$
\bar{\partial} g=\left.G\right|_{W_{\rho}(\Gamma)} \text {. }
$$

Proof. For the simplicity of notations, we only detail the dimension 2 case. The general case will be detailed in a forthcoming paper. Also, we assume the edge $M$ is straight, or simply $\mathbf{R}^{2}$. When $M$ is not straight, one can replace the involved wedges by suitable pseudo-wedges. We first consider the "if" part.

Note. We can assume that $u \in A^{\prime}\left(M_{\rho}\right)$ by restricting $u$ to $M_{\rho}$ because a Tsuno representation for a restriction of $u$ to $M_{\rho}$ differs from $G$ by a $\bar{\partial}$ closed form in $\mathbf{C}^{2} \backslash\left(X \backslash M_{\rho}\right)$, which is $\bar{\partial}$ exact in $M_{\rho}+i D_{\rho}$, where $D_{\rho}$ is the disk of radius $\rho$ and centered at 0 in $\mathbf{R}^{2}$, especially $\bar{\partial}$ exact in $W_{\rho}(\Gamma)$; thus, (5.1) is invariant under restriction.

Now, there is a wedge $W_{\rho}\left(\Gamma_{1}\right)$ with $\Gamma_{1}$ a strictly convex cone, $\sigma_{0} \cdot \Gamma_{1}<0$ and $\Gamma_{1} \cup \Gamma=\mathbf{R}^{2} \backslash\{0\}$. In this wedge, there is a smooth function $g_{1}$ so that

$$
\bar{\partial} g_{1}=\left.G\right|_{W_{\rho}\left(\Gamma_{1}\right)} \text {. }
$$

Notice that $\Gamma_{1} \cap \Gamma$ has two components $\Gamma_{1}^{\prime}$ and $\Gamma_{2}^{\prime}$, both of which are strictly convex. Let $h_{1}=g_{1}-\left.g\right|_{W_{\rho}\left(\Gamma_{1}^{\prime}\right)}, h_{2}=g-\left.g_{1}\right|_{W_{\rho}\left(\Gamma_{2}^{\prime}\right)}$, then they are holomorphic. It therefore follows from (4.1) and the Stokes' formula (the typical residue argument as in the proof of Theorem 4.2.1) that

$$
u \in \varepsilon\left[\left(B h_{1}\right)_{\Gamma_{1}^{\prime}}^{M_{\rho}}+\left(B h_{2}\right)_{\Gamma_{2}^{\prime}}^{M_{\rho}}\right]
$$

where $\varepsilon= \pm 1, \rho>0$. Thus, $\sigma_{0} \notin W F_{0}(u)$. Since we only consider the case in $C^{2}$, the proof of (5.2) is much easier than that of Theorem 4.2.1. For this reason, let us repeat the proof. Indeed, we can choose an arbitrarily small neighborhood $U_{\delta}$ of $M_{\rho} \quad\left(=D_{\rho}\right.$ in the present situation $)$ as follows,

$$
U_{\delta}=D_{\rho+\delta}+i D_{\delta} \text {. }
$$


Denote $\gamma_{t}=\{(r \cos t, r \sin t) \mid r>0\}, t \in \mathbf{R}$. By a rotation, we can assume that $\mathscr{C}=\left\{\gamma_{t} \mid-t_{1}<t<t_{1}\right\}, 0<t_{1}<\pi / 2$, is contained in $\Gamma_{1}$, and the interior (cone) of the compliment of $\mathscr{C}$ is contained in $\Gamma$. Note that $\partial U_{\delta}=$ $\left\{D_{\rho+\delta}+i C_{\delta}\right\} \cup\left\{C_{\rho+\delta}+i D_{\delta}\right\}$, where $C_{\delta}$ is the boundary of $D_{\delta}$. We now divide $\partial U_{\delta}$ into three pieces, i.e., $\partial U_{\delta}=S_{1} \cup S_{2} \cup S_{3}$, where

$$
\begin{gathered}
S_{1}=\partial U_{\delta} \cap W_{\rho}(\mathscr{C})=D_{\rho}+i \delta\left\{(\cos t, \sin t) \mid-t_{1}<t<t_{1}\right\}, \\
S_{2}=D_{\rho}+i \delta\left\{(\cos t, \sin t) \mid t_{1} \leq t \leq 2 \pi-t_{1}\right\},
\end{gathered}
$$

and $S_{3}$ is the compliment of $S_{1} \cup S_{2}$ in $\partial U_{\delta}$. Then, $S_{1} \subset W\left(\Gamma_{1}\right), S_{2} \subset$ $W(\Gamma), S_{1} \cup S_{2}=D_{\rho}+i C_{\delta}$, and $S_{1} \cap S_{2}=\Sigma_{1} \cup \Sigma_{2}$, where $\Sigma_{j}=M_{\rho}+i \delta \gamma_{j}$, $\gamma_{1}=\left(\cos t_{1}, \sin t_{1}\right), \gamma_{2}=\left(\cos \left(-t_{1}\right), \sin \left(-t_{1}\right)\right), j=1,2$. Suppose $W\left(\Gamma_{j}^{\prime}\right)$ is the component of $W(\Gamma) \cap W\left(\Gamma_{1}\right)$ containing $\Sigma_{j}$. While $S_{3}$ is contained in an arbitrarily small neighborhood of $\partial M_{\rho}$ as $\delta \rightarrow 0$. Give $\Sigma_{j}$ the orientation as the boundary of $S_{j}$. Note that the orientation of $\Sigma_{j}$ as the boundary of $S_{1}$ is opposite to that of $\Sigma_{1}$ as the boundary of $S_{2}$. From Stokes' formula, it follows that

$$
\begin{aligned}
\langle u, \psi\rangle & =\int_{\partial U_{\delta}} G(\zeta) \psi(\zeta) \wedge d \zeta \\
& =\int_{S_{1}} \bar{\partial} g_{1}(\zeta) \psi(\zeta) \wedge d \zeta+\int_{S_{2}} \bar{\partial} g(\zeta) \psi(\zeta) \wedge d \zeta+\int_{S_{3}} G(\zeta) \psi(\zeta) \wedge d \zeta \\
& =\int_{\Sigma_{1}}\left(g_{1}-g\right) \psi+\int_{\Sigma_{2}}\left(g-g_{1}\right) \psi+\int_{\partial S_{3}} \tilde{g} \psi+\int_{S_{3}} G(\zeta) \psi(\zeta) \wedge d \zeta \\
& =\int_{\Sigma_{1}} h_{1} \psi+\int_{\Sigma_{2}} h_{2} \psi+\int_{\partial S_{3}} \tilde{g} \psi+\int_{S_{3}} G(\zeta) \psi(\zeta) \wedge d \zeta
\end{aligned}
$$

where the integral over $\partial S_{3}$ is a boundary term from integrals over $S_{1}, S_{2}$ after using Stokes' formula. Notice that $u_{j \delta}: \psi \mapsto \int_{\Sigma_{j}} h_{j} \psi$ converges to $\varepsilon u_{j}$ for some $u_{j} \in\left(B h_{j}\right)_{\Gamma^{\prime}}^{M_{\rho}}$, where $\varepsilon= \pm 1$ depends only on the difference between the orientations of $\Sigma_{1}$ and $M_{\rho}$; and that the analytic functional defined by the other integrals (over $S_{3}$ or $\partial S_{3}$ ) converges to an analytic functional in $A^{\prime}\left(\partial M_{\rho}\right)$. Therefore, (5.2) is proved if we let $\delta \rightarrow 0$.

Now, we prove the "only if" part. Suppose $\sigma_{0} \notin W F_{0}(u)$. Then, there are $\rho>0$, and strictly convex $\Gamma_{j}$ together with holomorphic functions $h_{j}$ defined in $W_{\rho}\left(\Gamma_{j}\right), j=1, \ldots, l$, so that

(i) $M_{\rho} \subset X$,

(ii) $\sigma_{0} \cdot \Gamma_{j}<0$,

(iii) $\left.u\right|_{M_{\rho}}=\sum_{j=1}^{l}\left(B h_{j}\right)_{\Gamma_{j}}^{M_{\rho}}$.

From the note made in the proof of the "if" part, we see that it can be assumed that $u \in A^{\prime}\left(M_{\rho}\right)$ and there are $u_{j} \in\left(B h_{j}\right)_{\Gamma_{j}}^{M_{\rho}}$ so that

$$
u=\sum_{j=1}^{l} u_{j} .
$$

The proof can be further reduced to the case $l=1$, i.e., we are going to prove the following statement: 
Suppose $h$ is a holomorphic function in $W(\Gamma)$ and $u \in B h_{\Gamma}^{M_{\rho}}$, then any Tsuno representation form $G$ of $u$ is $\bar{\partial}$ exact in $W_{\rho}\left(\mathbf{R}^{2} \backslash \bar{\Gamma}\right)$.

In fact, we have a more precise version of this statement given later (see Lemma 5.3.1). Assume this statement for a moment. If $u$ satisfies (5.3) and $u_{j}$ has a Tsuno representation $G_{j}$, then we immediately see that $G=\sum_{j=1}^{l} G_{j}$ is a Tsuno representation for $u$. Since $G_{j}$ has been proved to be $\bar{\partial}$ exact in $\left.W_{\rho}\left(\mathbf{R}^{2}\right\rangle \bar{\Gamma}_{j}\right), G$ is $\bar{\partial}$ exact in $W_{\rho}(\Gamma)$, where $\Gamma=\mathbf{R}^{2} \backslash$ closed convex hull of $\bigcup_{j=1}^{l} \Gamma_{j}$. It is clear that $\Gamma$ is concave and $\sigma_{0} \cdot \partial \Gamma<0$. Since the other Tsuno forms differ from $G$ by a $\bar{\partial}$ exact form, the "only if" is proved.

Next, we prove the statement above.

Restricting ourselves in a slightly smaller cone, we can assume $h$ is defined in a larger wedge $W\left(\Gamma^{\prime}\right), \Gamma \Subset \Gamma^{\prime}$. By a rotation, we can further assume $\Gamma=$ $\left\{\gamma_{t} \mid-t_{0}<t<t_{0}\right\}$, with $0<t_{0}<\pi / 2$. Define a smooth function $g$ in $\mathbf{C}^{2} \backslash M_{\rho}$ so that $g=h$ on $W_{\rho}(\Gamma)$ and $g$ vanishes on the rays $M+i \gamma_{t}$ for $\pi / 2 \leq t \leq 3 \pi / 2$. Here we choose a very specific $g$. As mentioned in Remark 4.1.4, it could be arbitrarily changed outside of the cylinder $M_{\rho}+i D_{\rho}$ as long as it remains smooth for our purpose below. Since $h$ is holomorphic, $\bar{\partial} g=0$ on $W_{\rho}(\Gamma)$. We then construct a Tsuno representation form $G$ in the following way. Define

$$
G= \begin{cases}0, & \text { on } M_{\rho}+i \gamma_{t} \text { for } \pi \leq t \leq 2 \pi, \\ \bar{\partial} g, & \text { otherwise. }\end{cases}
$$

We now assert that $G$ is a Tsuno representation form of $\pm u$ modulo a $\bar{\partial}$ closed form in $\mathbf{C}^{2} \backslash \partial M_{\rho}$. Note that, in $W_{\rho}\left(\mathbf{R}^{2} \backslash \bar{\Gamma}\right), G=\bar{\partial} g_{1}$, where $g_{1}=g$ on $M_{\rho}+i \gamma_{t}$ for $0<t \leq \pi$ and $g_{1}=0$ on $M_{\rho}+i \gamma_{t}$ for $\pi<t<2 \pi$. The statement to be proved then follows from the fact that a $\bar{\partial}$ closed form in $C^{2} \backslash \partial M_{\rho}$ is also $\bar{\partial}$ exact in $D_{\rho}^{2}=D_{\rho}+i D_{\rho}$. To see that $G$ is a Tsuno representation form of $u$, we basically use the same argument as in the proof of (5.2). Choose a neighborhood $U_{\delta}$ of $M_{\rho}$ as before, and write $\partial U_{\delta}=S \cup S^{\prime}$, where $S=$ $M_{\rho}+i C_{\delta}, S^{\prime}$ is the compliment of $S$ in $\partial U_{\delta}$. Let $\Sigma=M_{\rho}+i(\delta, 0) \quad(\subset S)$ be given the orientation of the boundary of $S \backslash \Sigma$. Then, for any holomorphic function $\psi$,

$$
\begin{aligned}
\int_{\partial U_{\delta}} G(\zeta) \psi(\zeta) \wedge d \zeta & =\int_{S} G(\zeta) \psi(\zeta) \wedge d \zeta+\int_{S^{\prime}} G(\zeta) \psi(\zeta) \wedge d \zeta \\
& =\int_{S \backslash \Sigma} \bar{\partial} g_{1} \psi+\int_{S^{\prime}} G(\zeta) \psi(\zeta) \wedge d \zeta \\
& =\int_{\Sigma} g \psi+\int_{C_{\rho}+i C_{\delta}} g_{1} \psi+\int_{S^{\prime}} G(\zeta) \psi(\zeta) \wedge d \zeta
\end{aligned}
$$

Letting $\delta \rightarrow 0$, we have the analytic functional $\psi \mapsto \int_{\Sigma} h \psi$ converges to $\pm u$, where \pm depends only on the orientation of $\Sigma$, and the analytic functional defined by the other integrals in the last line above converges to an analytic functional in $A^{\prime}\left(\partial M_{\rho}\right)$. Therefore, $G$ is a Tsuno form of $\pm u$ modulo a $\bar{\partial}$ closed form in $C^{2} \backslash \partial M_{\rho}$ (see Remark 4.1.3). 
5.3. Precise description of wave front by Tsuno forms, holomorphic extension, and monodromy. Next, we only consider the situation in $\mathbf{C}^{2}$. We are still lacking a good understanding of higher dimension case. We want to investigate the case when a "cone" $\Gamma$ is not in the wave front set (so far we just considered a ray). In two variables, if a closed cone of aperture $\theta$ is not in the wave front set, it will be shown that the equation $\bar{\partial} g=G$ ( $G$ a Tsuno form) can be solved on a corresponding "wedge" of aperture $\theta+\pi$. If $\theta<\pi$, the wedge is just in the usual sense. If $\theta>\pi$, then it should be understood in the universal covering (thus monodromy phenomenon does occur, see Corollary 5.3.1). To demonstrate the monodromy phenomenon in $\mathbf{C}^{2}$, we introduce some notations. Let $\widehat{\mathbf{R}^{2}}=:\left\{\left(\gamma_{t}, t\right) \in \mathbf{R}^{3} \mid t \in \mathbf{R}\right\}$ be the universal covering of $\mathbf{R}^{2} \backslash\{0\}$, where $\gamma_{t}=\{(r \cos t, r \sin t) \mid r>0\}$ as before, and let $\mathbf{p}: \widehat{\mathbf{R}^{2}} \rightarrow \mathbf{R}^{2} \backslash\{0\}$ be the canonical projection.

Definition 5.3.1. (i) A set in $\widehat{\mathbf{R}^{2}}$ of the form $\Gamma=\Gamma_{(a, b)}=:\left\{\left(\gamma_{t}, t\right) \mid a<t<b\right\}$, for some $a<b$, is called a lifted cone. Meantime, $W_{\rho}(\Gamma)=\left\{\left(z+i \gamma_{t}, t\right) \mid z \in\right.$ $\left.M_{\rho}, a<t<b\right\}$ is called a lifted wedge.

(ii) Let $g$ be a smooth function on a lifted wedge $W_{\rho}\left(\Gamma_{(a, b)}\right)$, for each direction $\gamma_{t_{0}}, a<t_{0}<b$, we define a local projection $g_{t_{0}}^{*}$ of $g$ by setting

$$
g_{t_{0}}^{*}\left(z+i \gamma_{t}\right)=g\left(z+i \gamma_{t}, t\right)
$$

with $t$ near $t_{0}$. A lifted cone $\Gamma_{(a, b)}$ is called a solvable cone for a $\bar{\partial}$ closed $(0,1)$ form $G$ defined on $\mathbf{C}^{2} \backslash M$, if there is a smooth function $g$ defined on $W_{\rho}\left(\Gamma_{(a, b)}\right)$ for some $\rho>0$ so that, for each $t \in(a, b)$, the local projection $g_{t}^{*}$ satisfies

$$
\bar{\partial} g_{t}^{*}=G
$$

near the direction of $\gamma_{t}$.

The following lemma gives an exact description of the wave front set in terms of the solvable cones.

Lemma 5.3.1. Let $\Gamma=\left\{\gamma_{t} \mid a<t<b\right\}$ be a component of the set $\mathbf{R}^{2} \backslash W F_{0}(u)$, where $b \leq a+2 \pi$ if $\Gamma \neq \mathbf{R}^{2}-\{0\}$ or $a=-\infty, b=\infty$ otherwise. Set

$$
\Gamma^{*}=\Gamma_{(a, b)}^{*}=:\left\{\left(\gamma_{t}, t\right) \in \widehat{\mathbf{R}^{2}} \mid a-\pi / 2<t<b+\pi / 2\right\} .
$$

Then, $\Gamma^{*}$ is a maximal solvable cone $\Gamma^{*}\left(\subset \widehat{\mathbf{R}^{2}}\right)$ for the Tsuno representation form $G$ of $u$ in the following sense: for any cone $\Gamma_{1} \Subset \Gamma^{*}, \Gamma_{1}$ is solvable; for any cone $\Gamma_{1}$ containing $\Gamma, \Gamma_{1}$ is not solvable.

As an immediate consequence of this lemma, we can visualize some interesting phenomenon of holomorphic extension for analytic functionals.

Corollary 5.3.1. Let $G$ be a Tsuno form of $u \in A^{\prime}(X)$ with $X$ as before. We have

(i) If $\bar{\partial} g=G$ is solvable on a lifted wedge of aperture $>2 \pi$, in other words, the wavefront of $u$ is contained in a strictly convex cone $\Gamma$, then, $u$ can be holomorphically extended to the wedge $W_{\rho}\left(\Gamma^{0}\right)$, where $\Gamma^{0}=\left\{\sigma \in \mathbf{R}^{2} \mid \sigma \cdot \gamma \geq 0\right.$, $\forall \gamma \in \Gamma\}$.

(ii) If $\bar{\partial} g=G$ is solvable on a lifted wedge of aperture $>3 \pi$, i.e., $W F_{0}(u)=$ $\varnothing$, then $u$ can be holomorphically extended to a neighborhood of 0 . 
Proof of Lemma 5.3.1. For any direction $\gamma_{t} \in \Gamma, a<t<b$. Then, $\gamma_{t} \notin$ $W F_{0}(u)$ implies that there is a solvable cone $\Gamma_{t}=: \Gamma_{\left(t-\pi / 2-\delta_{t}, t+\pi / 2+\delta_{t}\right)}$, where $\delta_{t}>0$, and a smooth function $g_{t}$ in $W_{\rho_{t}}\left(\Gamma_{t}\right)$ satisfying (5.4). It is clear that $\Gamma_{t} \subset \Gamma^{*}$. For otherwise, we could have a holomorphic decomposition of $u$ which contradicts to the fact that $\Gamma$ is a component (there would be a conic neighborhood of the direction $\gamma_{b}$ not in $W F_{0}(u)$ if $t+\delta_{t}>b$, or that of $\gamma_{a}$ if $\left.t-\delta_{t}<a\right)$, by calculating the residue of $G$ as in the proof of (5.2).

We now consider the relation between two such solvable cones.

Sublemma 5.3.1. If $\Gamma_{t_{1}}, \Gamma_{t_{2}}$ are two concave solvable cones for $G$ such that $\Gamma_{t_{1}} \cap \Gamma_{t_{2}}$ is also concave, then $\Gamma_{t_{1}} \cup \Gamma_{t_{2}}$ is also solvable for $G$.

Assume this sublemma for a moment, let us continue the proof of the lemma.

Consider any closed cone $\mathscr{C} \Subset \Gamma^{*}$. Suppose $\mathscr{C}$ is concave, otherwise $\mathscr{C}$ is certainly solvable for $G$ due to the convexity. $\mathscr{C}=\left\{\gamma_{t} \in \mathbf{R}^{2} \backslash\{0\} \mid c-\pi / 2 \leq t \leq\right.$ $d+\pi / 2\}$ for some $a<c \leq d<b$. Now, we have the following open covering of the interval $[c, d]:\left\{\left(t-\delta_{t} / 2, t+\delta_{t} / 2\right)\right\}_{t \in[c, d]}$. Therefore, a finite number of these open intervals can cover $[c, d]$, i.e., there are numbers $t_{1}<t_{2}<\cdots<t_{q}$ in $[c, d]$ so that $\bigcup_{j=1}^{q}\left(t_{j}-\delta_{t_{j}} / 2, t_{j}+\delta_{t_{j}} / 2\right) \supset[c, d]$. This implies that $t_{j+1}-$ $t_{j}<\left(\delta_{j}+\delta_{j+1}\right) / 2$. Note that $\Gamma_{t_{j}} \cap \Gamma_{t_{j+1}}=\left\{\gamma_{t} \mid t_{j+1}-\pi / 2-\delta_{t_{j+1}}<t<t_{j}+\pi / 2+\delta_{t_{j}}\right\}$ has aperture $\pi+\delta_{t_{j}}+\delta_{t_{j+1}}-\left(t_{j+1}-t_{j}\right)>\pi$. Hence, it is concave. Therefore, a consecutive use of the sublemma above yields that $\mathscr{C}$ is a solvable cone for $G$. The lemma is then proved.

Proof of Sublemma 5.3.1. Let $g_{1}, g_{2}$ be solutions of the equation $\bar{\partial} g=G$ respectively in $W_{\rho}\left(\Gamma_{t_{1}}\right), W_{\rho}\left(\Gamma_{t_{2}}\right)$. Note that the projection of the function $h=$ $g_{1}-g_{2}$ is holomorphic in a concave wedge $W_{\rho}\left(\Gamma_{t_{1}} \cap \Gamma_{t_{2}}\right)$ by the hypothesis in the sublemma. By Bochner's tube theorem, $h$ is actually a lift of a holomorphic function defined in a neighborhood of 0 . Define

$$
\tilde{g}= \begin{cases}g_{1}, & \text { on } W_{\rho^{\prime}}\left(\Gamma_{t_{1}}\right), \\ g_{2}+h, & \text { on } W_{\rho^{\prime}}\left(\Gamma_{t_{2}}\right) \text { for some } \rho^{\prime}>0 .\end{cases}
$$

Then, $\tilde{g}$ is a well-defined smooth function on $W_{\rho^{\prime}}\left(\Gamma_{t_{1}} \cup \Gamma_{t_{2}}\right)$ and satisfies (5.4). This finishes the proof of the sublemma.

Because there may be many components in $\mathbf{R}^{2} \backslash W F_{0}(u)$, we can have the same amount of maximal solvable cones associated with a Tsuno representation form $G$ of $u$. Conversely, these maximal solvable cones determine all the components of $\mathbf{R}^{2} \backslash W F_{0}(u)$ and hence $W F_{0}(u)$ completely.

We now show Corollary 5.3.1.

Proof of Corollary 5.3.1. Proof of (i). $W F_{0}(u) \subset \Gamma$ implies that there is a component of $\left.\mathbf{R}^{2} \backslash \overline{W F_{0}(u)} \supset \supset \mathbf{R}^{2}\right\rangle \bar{\Gamma}$. Thus, at least $\left.\left(\mathbf{R}^{2}\right\rangle \bar{\Gamma}\right)^{*}$ is a solvable cone for $G$ from Lemma 5.3.1. Note that if $\Gamma=\left\{\gamma_{t} \mid a<t<b\right\}$, then $\left(\mathbf{R}^{2} \mid \bar{\Gamma}\right)^{*}=$ $\left\{\left(\gamma_{t}, t\right) \mid b-\pi / 2<t<a+5 \pi / 2\right\}$. Therefore, it is clear that the equation $\bar{\partial} g=G$ is solvable in a lifted wedge of aperture $>2 \pi$ if and only if the wavefront of $u$ is contained in a strictly convex cone. Notice that the overlapped portion of the shadow of $\left(\mathbf{R}^{2} \mid \bar{\Gamma}\right)^{*}$ under the canonical projection $\mathbf{p}$ onto $\mathbf{R}^{2}$ is exactly $\Gamma^{0}$. Let $g$ be a smooth function on $W_{\rho}\left(\left(\mathbf{R}^{2} \backslash \bar{\Gamma}\right)^{*}\right)$ satisfying the solvability condition (5.2), and let $g_{1}, g_{2}$ be the two local projections of $g$ on $W_{\rho}\left(\Gamma^{0}\right)$ 
defined respectively by

$$
\begin{aligned}
& g_{1}\left(z+i \gamma_{t}\right)=g\left(z+i \gamma_{t}, t\right), \\
& g_{2}\left(z+i \gamma_{t}\right)=g\left(z+i \gamma_{t+2 \pi}, t+2 \pi\right), \quad b-\pi / 2<t<a+\pi / 2, z \in M_{\rho} .
\end{aligned}
$$

Then, $f=g_{2}-g_{1}$ is obviously holomorphic on $W_{\rho}\left(\Gamma^{0}\right)$. By calculating the residue of $G$ in the way shown in the proof of (5.2), we see that $\left.u\right|_{M_{\rho}}$ is the boundary value of $f$.

The proof of (ii) is basically the same.

Next, we prove a hyperfunction version of the edge of the wedge theorem in $\mathbf{C}^{m}$ to demonstrate the holomorphic extension in higher dimension spaces. Martineau's cohomological method is used in the following proof. Another method is using the FBI transform (see next section).

Theorem 5.3.1. Let $\Gamma$ be a strictly convex cone in $\mathbf{R}^{m}$ with vertex 0 . If $f$ is holomorphic in $W_{\rho}(\Gamma) \cup W_{\rho}(-\Gamma)$ and $B f_{\Gamma}^{M_{\rho}}=B f_{-\Gamma}^{M_{\rho}}$ in the hyperfunction sense, then $f$ can be holomorphically extended to a neighborhood of 0 .

Proof. Without loss of generality, we assume $W_{\rho}(\Gamma)=U_{1, \ldots, m}, W_{\rho}(-\Gamma)=$ $U_{-1, \ldots,-m}$ by using the notion defined in $\S 2.1$. Consider the cocycle $\left\{f_{j_{1}}, \ldots, j_{m}\right\} \in$ $C^{m-1}(\mathscr{U}, \mathscr{O})$ with

$$
\begin{aligned}
f_{1, \ldots, m} & =\left.f\right|_{U_{1, \ldots, m}}, \\
f_{-1, \ldots,-m} & =\left.(-1)^{m+1} f\right|_{U_{-1, \ldots,-m}}, \\
f_{j_{1}, \ldots, j_{m}} & =0, \quad \text { otherwise. }
\end{aligned}
$$

Then, $\left\{f_{j_{1}}, \ldots, j_{m}\right\}$ is cohomologous to 0 in a slightly smaller neighborhood of 0 from the hypothesis in the theorem. Once this is clear, the induction argument on the dimension $m$ in [M3] (see also [P, pp. 452-455] without deleting a cubic neighborhood) applies to give a holomorphic extension of $f$ to a neigborhood of 0 . Note that the case $m=2$ is already proved before the statement of the theorem since $B f_{\Gamma}^{M_{\rho}}=B f_{-\Gamma}^{M_{\rho}}$ implies the wave front set of $f$ is empty.

5.4. The FBI transform and wave front set. We now consider the wave front set from the point of view of the FBI transform (see [BCT, BR1, BR2, BRT]). The FBI transform of an analytic functional is defined as follows.

Definition 5.4.1. The FBI transform of $u \in A^{\prime}(X)$ with $X$ as before, denoted by $F(u ; w, \sigma)$, is defined by

$$
F(u ; w, \sigma)=u\left(e^{i \sigma(w-\cdot)-\langle\sigma\rangle(w-\cdot)^{2}} \Delta(w-\cdot, \sigma)\right),
$$

where $\sigma, w \in \mathbf{C}^{m}$,

$$
\Delta(w, \sigma)=\operatorname{det} \frac{\partial \theta}{\partial \sigma}(w, \sigma), \quad \theta=\sigma+i\langle\sigma\rangle w, \quad\langle\sigma\rangle=\left(\sum_{j=1}^{m} \sigma_{j}^{2}\right)^{1 / 2} .
$$

This definition extends the corresponding one in [BR2] if $u$ is taken to be the boundary value of a holomorphic function in a wedge. In fact, in view of the Decomposition Theorem 2.3.1 and the argument in [BR2], we can still prove the following characterization of the wave front set. 
Theorem 5.4.1. Let $u$ be as above. Then, $\sigma_{0} \notin W F_{0}(u)$ if and only if there exist constants $c, C, \tau>0$ so that

$$
|F(u ; w, \sigma)| \leq C e^{-c|\sigma|},
$$

for $w \in \mathbf{C}^{m},|w|<\tau$, and $\sigma \in \mathscr{C}$, a conic neighborhood of $\sigma_{0}$ in $\mathbf{C}^{m} \backslash\{0\}$.

Sketch of proof. By Theorem 4.2.1, there is a holomorphic decomposition $h_{j} \in$ $A\left(W_{\rho}\left(\Gamma_{j}\right)\right), j=1, \ldots, l$, of $u$ so that

(i) $\sigma_{0} \in \Gamma_{1}, \sigma_{0} \cdot \Gamma_{j}<0, \forall j>1$;

(ii) $\left.u\right|_{M_{\rho}}=\sum_{j=1}^{l}\left(B h_{j}\right)_{\Gamma_{j}}^{M_{\rho}}$, for some small $\rho>0$.

Note that $\sigma_{0} \cdot \Gamma_{j}<0, j>1$, implies that (5.4) holds for any $u_{j} \in\left(B h_{j}\right)_{\Gamma_{j}}^{M_{\rho}}$, $j>1$. Therefore, the proof is reduced to proving $\sigma_{0} \notin W F_{0}\left(\left(B h_{1}\right)_{\Gamma_{1}}^{M_{\rho}}\right)$ if and only if (5.4) holds for any $u_{1} \in\left(B h_{1}\right)_{\Gamma_{1}}^{M_{\rho}}$. This follows from Theorem 1 in [BR2].

We also mention that the edge of the wedge theorem (Theorem 5.3.1) can also be proved in terms of FBI transform by applying Theorem 2 in [BR2]. Nevertheless, we are still unable to extend the holomorphic extension result such as Corollary 5.3.1 to higher dimension spaces by the FBI transform method due to the lack of the convergence of the inverse FBI transform in the analytic functional sense.

\section{SOME REMARKS ABOUT HYPERFUNCTIONS, DISTRIBUTIONS, AND $\bar{\partial}$ PROBLEM WITH PARTIAL COMPACT SUPPORT}

6.1. Flabbiness of the sheaf of germs of hyperfunctions. To any relatively compact set $X \subset M$, a $C^{1}$ totally real manifold, there is associated a space $B(X) \quad(=B(\bar{X}))$, as in Definition 3.2.1, equipped with the induced topology from the $w^{*}$-topology in $A^{\prime}(X)$. When $M$ is real analytic, or simply $M=$ $\mathbf{R}^{m}, X \mapsto B(X)$ defines a flabby sheaf from the standard hyperfunction theory (refer to $[\mathrm{SKK}]$ or [Sch]). This fact was generalized to a totally real manifold by Harvey and Wells [Hv1, HvWe]. Rather than using the sheaf cohomology technique, we can make use of the explicit duality formula (4.1) to show the flabbiness of the sheaf of hyperfunctions defined here at an elementary level as Hörmander treated in [H1]. This approach gives us additional interests such as the convergence of a sequence of analytic functionals and some relation with solving $\bar{\partial}$ problems, etc.

In this section, we assume that $M$ satisfies the following property: any compact subset of $M$ is polynomially convex, and $M$ is countable at infinity. Note that, in general, a totally real manifold only has this property locally.

The following decomposition theorem of analytic functionals due to Martineau [M2] will yield the existence of the restriction map.

Theorem 6.1.1. Let $u \in A^{\prime}(X), X \Subset M$. If $X=\bigcup_{j=1}^{k} X_{j}, X_{j} \Subset M$, then there exist $u_{j} \in A^{\prime}\left(X_{j}\right), j=1, \ldots, k$, so that $u=\sum_{j=1}^{k} u_{j}$, where we view $u_{j}$ as an element of $A^{\prime}(X)$ because of the obvious injection $i: A^{\prime}\left(X_{j}\right) \rightarrow A^{\prime}(X)$.

Lemma 6.1.1. Let $V_{0}$ and $V$ be two compact and polynomial convex sets with $V_{0} \subset \operatorname{int}(V) \subset \mathbf{C}^{m}$. For any $\bar{\partial}$ closed $(0, m-1)$ form $K$ defined in $\mathbf{C}^{m} \backslash V$, 
and for any $\varepsilon, R>0$, there is a $\bar{\partial}$ closed $(0, m-1)$ form $K_{\varepsilon}$ defined in $\mathbf{C}^{m} \backslash V_{0}$ so that

$$
\left\|K_{\varepsilon}-K\right\|_{C^{0}\left(B_{R}^{m} \backslash V\right)}<\varepsilon,
$$

where $B_{R}^{m}=\left\{z \in \mathbf{C}^{m}|| z \mid \leq R\right\}$.

Proof. It suffices to consider the case $\operatorname{int}\left(V_{0}\right) \neq \varnothing$ by exhausting successively. The following argument is heritated from the standard proof of the Runge approximation theorem. Extend continuously the $\bar{\partial}$ closed form $K$ to $\mathbf{C}^{m}$, denoted by $\widehat{K}$. Then, by the integral representation formula (see, for example, [HL]) in $B_{R}^{m}$,

$$
\widehat{K}(z)=\bar{\partial}(T(\widehat{K}))(z)+\int_{V} \bar{\partial} \widehat{K}(\zeta) \wedge B M_{m-1}(\zeta, z) \wedge d \zeta,
$$

where $B M_{m-1}(\zeta, z)$ is the $(m-1)$ th Bochner-Martinelli kernel. Therefore, it is enough to show

$$
\overline{\operatorname{span}_{\zeta \in V}\left\{B M_{m-1}(\zeta, \cdot)\right\}}=\overline{\operatorname{span}_{\zeta \in V_{0}}\left\{B M_{m-1}(\zeta, \cdot)\right\}},
$$

where the closure is in the topology of $C^{0}\left(B_{R}^{m} \backslash V\right)$. It is equivalent, by duality, to show that

$$
\mu(\zeta)=: \int_{B_{R}^{m} \backslash V} \omega(z) \wedge B M_{m-1}(\zeta, z)=0, \quad \forall \zeta \in V_{0},
$$

for some $(m, 1)$ current $\omega(z)$ implies

$$
\int_{B_{R}^{m} \backslash V} \omega(z) \wedge B M_{m-1}(\zeta, z)=0, \quad \forall \zeta \in V .
$$

Note that

$$
B M_{m-1}(\zeta, z)=c_{m-1} \sum_{j=1}^{m}(-1)^{j-1} \frac{\left(\bar{\zeta}_{j}-\bar{z}_{j}\right)}{|\zeta-z|^{2 m}} d \bar{z}_{1} \wedge \cdots \wedge \widehat{d \bar{z}}_{j} \wedge \cdots \wedge d \bar{z}_{m}
$$

is real analytic, therefore, so is $\mu(\zeta)$ for $\zeta \in \operatorname{int}(V)$. Since $\operatorname{int}\left(V_{0}\right) \neq \varnothing$, $\mu(\zeta) \equiv 0$ in $\operatorname{int}(V)$, which completes the proof.

We can now extend the convergence theorem for a sequence of analytic functionals in [H1, Theorem 9.1.7] onto a totally real manifold. The proof of the following theorem is basically the same as Hörmander's except we replace the Runge approximation theorem for harmonic functions by Lemma 6.1.1, and the harmonic representation by Tsuno representation.

Theorem 6.1.2. Let $X_{0}$ and $X$ be compact sets with $X_{0} \subset X \subset M$, and let $u_{j} \in A^{\prime}\left(\mathbf{C}^{m}\right)$. Also, assume that

(i) for any compact neighborhood $V$ of $X$ in $\mathbf{C}^{m}$, we have $u_{j} \in A^{\prime}(V)$ for large $j$;

(ii) for any compact neighborhood $V_{0}$ of $X_{0}$ in $\mathbf{C}^{m}$, we have $u_{j}-u_{k} \in A^{\prime}\left(V_{0}\right)$ for large $j, k$. Then, there is $u \in A^{\prime}(X)$ so that, for any compact neighborhood $V_{0}$ of $X_{0}, u_{j}-u \in A^{\prime}\left(V_{0}\right)$ for large $j$, and $u$ is unique up to an element in $A^{\prime}\left(X_{0}\right)$.

Definitely, one of the immediate consequences is the existence of the support of an analytic functional carried on a totally real manifold as in [H1]. Another 
important consequence is the flabbiness of the sheaf of germs of hyperfunctions on a $C^{1}$ totally real manifold. The flabbiness of the sheaf of germs of hyperfunctions plays a fundamental role in the evolution of the hyperfunction theory.

6.2. Distribution boundary value and decomposition. This section discusses the relation between the hyperfunction boundary value in $\S 3$ and the usual distribution boundary values of holomorphic functions with slow growth, and to give a different proof of the Baouendi-Chang-Treves decomposition theorem based on the idea in the paper.

To define distribution, we assume that $M$ is $C^{\infty}$ smooth in this section and restrict ourselves to a neighborhood $M_{\rho}$ of 0 . We then define the distribution boundary value $B f_{\Gamma}$ of a holomorphic function $f$ defined in $W_{\rho}(\Gamma)$ as follows.

$$
\left\langle B f_{\Gamma}, \psi\right\rangle=\lim _{\substack{\gamma \in \Gamma \\|\gamma| \rightarrow 0}} \int_{M} f(w+i \gamma) \psi(w) d w, \quad \forall \psi \in C_{0}^{\infty}\left(M_{\rho}\right),
$$

if $f$ has polynomial growth near the edge $M$, i.e., for any $K \Subset M_{\rho}$, there is $k \in \mathbf{N}$ so that

$$
|f(z)| \leq \frac{C}{[\operatorname{dist}(z, M)]^{k}}, \quad \forall z \in K+i \Gamma_{\rho} .
$$

Now, suppose $B f_{\Gamma}$ exists and $X$ is a compact neighborhood of 0 in $M_{\rho}$. Let $\chi$ be a cutoff function in $C_{0}^{\infty}\left(M_{\rho}\right)$ such that $\chi=1$ on $X$. Then, we have

Proposition 6.2.1. There is a restriction of $\chi\left(B f_{\Gamma}\right)$ on $X$ which belongs to $B f_{\Gamma}^{X}$ in the hyperfunction sense.

Proof. The proof is straightforward. One needs only to verify that for any neighborhood $V$ of $K=\overline{\operatorname{supp}(\chi) \backslash X}$, and for any $\psi \in A\left(\mathbf{C}^{m}\right)$,

$$
\left|\left(\chi\left(B f_{\Gamma}\right)-u^{X}\right)(\psi)\right| \leq C_{V}\|\psi\|_{L^{\infty}(V)},
$$

where $u^{X}$ is a representative in $B f_{\Gamma}^{X}$ as in Theorem 3.2.1, $C_{V}$ is a constant independent of $\psi$. (6.2) follows from the following consideration. Choose $\gamma$ small so that $K+i \gamma \subset V_{1}$ and $u_{\gamma}^{X}-u^{X} \in A^{\prime}\left(V_{1}\right)$ with $\partial_{M} X \subset V_{1} \Subset V$. Then,

$$
\begin{aligned}
\left(\chi B f_{\Gamma}-u^{X}\right)(\psi)= & \left(u_{\gamma}^{X}-u^{X}\right)+\int_{K+i \gamma} \chi(w) f(w) \psi(w) d w \\
& +\iint_{K+i(0, \gamma]} \bar{\partial} \chi \wedge(f \psi d w) .
\end{aligned}
$$

Obviously, the first and the second terms on the right-hand side are in $A^{\prime}\left(V_{1}\right)$; while it is almost standard that if $f$ has polynomial growth near the edge $M$, then $\psi \mapsto \iint_{K+i(0, \gamma]} \bar{\partial} \chi \wedge(f \psi d w)$ defines a distribution with support $K+$ $i[0, \gamma]$, hence, $\left(\chi B f_{\Gamma}-u_{X}\right) \in A^{\prime}\left(V_{1}\right)$.

We now follow the analytic functional argument in $\S 4.2$ to give another proof of the Baouendi-Chang-Treves decomposition theorem based on a certain $\bar{\partial}$ regularity result cited in $\S 2.1$ in the distribution case. Let $u$ be a distribution with compact support $X$ on $M$. Assume $X$ is a compact neighborhood of $0 \subset \Omega$. As mentioned before, $G(\zeta)=\langle u, B M(\cdot, \zeta)\rangle$ is a Tsuno representation 
form of $u$. More importantly, $G(\zeta)$ has polynomial growth near the edge $X$. We can assume that

$$
|G(\zeta)| \leq \frac{C}{[\operatorname{dist}(\zeta, X)]^{k}}
$$

In $U_{j}$, put $\varphi_{j}(\zeta)=-k \ln \left(\operatorname{dist}\left(\zeta, \partial U_{j}\right)\right)$, then $G_{j} \in L^{2}\left(U_{j}, \varphi_{j}\right)$ as defined in 2.1. Consequently, Hörmander's $L^{2}$ estimate with weight $\varphi_{j}$ (Theorem 2.1.1) yields the existence of $G_{j_{1} \cdots j_{p}} \in L^{2}\left(U_{j_{1} \cdots j_{p}}, \varphi_{j_{1} \cdots j_{p}}\right)$ with $\varphi_{j_{1} \cdots j_{p}}=\sum_{s=1}^{p} \varphi_{j_{q}}$. In particular, we obtain a holomorphic cocycle $\left\{G_{j_{1} \cdots j_{m}}\right\}$ with $G_{j_{1} \cdots j_{m}} \in$ $L^{2}\left(U_{j_{1} \cdots j_{m}}, \varphi_{j_{1} \cdots j_{m}}\right)$. Possibly by shrinking the "wedge" $U_{j_{1} \cdots j_{m}}$ with the edge fixed, one can find that $G_{j_{1} \cdots j_{m}}$ has polynomial growth near the edge $X$ since $G_{j_{1} \cdots j_{m}}$ is holomorphic. Then, Theorem 4.2.1 shows that

$$
\left.u\right|_{X} \in \varepsilon \sum_{\substack{j_{p}= \pm p \\ p=1, \ldots, m}} \operatorname{sgn}\left(j_{1} \cdots j_{m}\right)\left(B G_{j_{1} \cdots j_{m}}\right)_{U_{j_{1}} \cdots j_{m}}^{X}
$$

in the hyperfunction sense. Possibly by shrinking the neighborhood $X$ to $X_{1}$, we see that $u$ coincides with

$$
\varepsilon \sum_{\substack{j_{p}= \pm p \\ p=1, \ldots, m}} \operatorname{sgn}\left(j_{1} \cdots j_{m}\right)\left(B G_{j_{1} \cdots j_{m}}\right)_{U_{j_{1}} \cdots j_{m}}
$$

in the distribution sense from Proposition 6.2.1 and the density of holomorphic functions in the space of smooth functions locally on a totally real manifold.

6.3. $\bar{\partial}$ problem with partial compact support in a cylinder. We study the problem of solving $\bar{\partial}$ equation with partial compact support in the domain $\left\{x \in \mathbf{R}^{2},|x|<1\right\}+i\left\{x \in \mathbf{R}^{2}, 0<|x| \leq 1\right\}$. In particular, a solvability criterion of the Greiner-Kohn-Stein type is provided.

Let $D_{2}=\left\{x \in \mathbf{R}^{2},|x| \leq 1\right\}, \widetilde{D}_{2}=\left\{x \in \mathbf{R}^{2}, 0<|x| \leq 1\right\}$.

Lemma 6.3.1. Let $g$ be a $\bar{\partial}$ closed smooth $(0,1)$ form in $D_{2}+i \widetilde{D}_{2}$. Then, $g$ is $\bar{\partial}$ exact if and only if

the analytic functional defined by $\psi \mapsto \int_{S_{\delta}} g \psi$ is carried by $S_{\delta}^{\prime}$, $\forall 0<\delta<1$,

where $S_{\delta}=D_{2}+i\{|x|=\delta\}, S_{\delta}^{\prime}=\partial D_{2}+i \delta D_{2}$ are defined in Remark 4.1.4.

Proof. Necessity is trivial by Stokes' theorem. We now prove the sufficiency. Denote $T_{\delta}=\partial D_{2}+B_{\delta}$, where $B_{\delta}=\left\{z \in \mathbf{C}^{2},|z|<\delta\right\}$. This is a toruslike neighborhood of $\partial D_{2}$. We first construct a $\bar{\partial}$ closed smooth extension $g_{\delta}$ of $g$ to $\mathbf{C}^{2} \backslash\left(D_{2} \cup T_{\delta}\right)$ in the sense that $g_{\delta}$ coincides with $g$ on the set $\Omega_{\delta}=(1-\delta) D_{2}+i D_{2}$. Extend $g$ smoothly to $\mathbf{C}^{2} \backslash D_{2}$. Notice that $\bar{\partial} g$ extends smoothly to $\mathbf{C}^{2} \backslash T_{\delta}$, it is also a top degree form. Then, there is a $(0,1)$ form $g_{1}$ on $\mathbf{C}^{2}-T_{\delta}$ so that $\bar{\partial} g_{1}=\bar{\partial} g$; hence, $g-g_{1}$ is a $\bar{\partial}$ closed $(0,1)$ form on $\mathbf{C}^{2} \backslash\left(D_{2} \cup T_{\delta}\right)$. Since $\bar{\partial} g_{1}=0$ in $\Omega_{\delta}, g_{1}$ is exact in $\Omega_{\delta}$, i.e., there is a smooth $g_{2}$ so that $\bar{\partial} g_{2}=g_{1}$ in $\Omega_{\delta}$. Smoothly extend $g_{2}$ to $\mathbf{C}^{2}$. Then, $g_{\delta}=g-g_{1}+\bar{\partial} g_{2}$ is $\bar{\partial}$ closed in $\mathbf{C}^{2} \backslash\left(X \cup T_{\delta}\right)$ and $g_{\delta}=g$ on $\Omega_{\delta}$.

For any neighborhood $U$ of $D_{2} \cup T_{\delta}$, it can be verified that the analytic functional $u_{\delta}: \psi \mapsto \int_{\partial U} g_{\delta} \psi$ is carried by $T_{\delta}$ from the assumption (6.4) of the lemma. Let $G_{\delta}$ be a Tsuno form of $u_{\delta}$ defined in $C^{2} \backslash T_{\delta}$, then $G_{\delta}-g_{\delta}$ satisfies 
$\int_{\partial U}\left(G_{\delta}-g_{\delta}\right) \psi=0$ for any holomorphic function $\psi$ and any neighborhood $U$ of $D_{2} \cup T_{\delta}$, and hence for any neighborhood $U$ of $P_{\delta}=D_{2}+B_{\delta}$. Hence, it follows from Lemma 4.1.1 that $G_{\delta}-g_{\delta}$ is $\bar{\partial}$ exact in $\mathbf{C}^{2} \backslash P_{\delta}$ since $P_{\delta}$ is convex. Since $G_{\delta}$ is $\bar{\partial}$ exact in $\Omega_{\delta}, g_{\delta}$ is $\bar{\partial}$ exact in $\Omega_{\delta}^{\prime}=\Omega_{\delta} \backslash P_{\delta}$, and so is the form $g$.

Choose a decreasing sequence of positive numbers $\left\{\delta_{n}\right\}_{n=1}^{\infty}, \delta_{n} \rightarrow 0$ as $n \rightarrow$ $\infty$. From the above argument, there is a smooth function $f_{n}$ so that $\bar{\partial} f_{n}=g$ on $\Omega_{\delta_{n}}^{\prime}$. Then, $h_{n}=f_{n+1}-f_{n}$ is holomorphic in $\Omega_{\delta_{n}}^{\prime}$. By Bochner's tube theorem, $h_{n}$ can be holomorphically extended to $\Omega_{\varepsilon_{n}}$ for some $\varepsilon_{n}>0, \varepsilon_{n} \rightarrow 0$ as $n \rightarrow 0$. To construct an exact solution of the equation $\bar{\partial} f=g$ in $\Omega \backslash D_{2}$, we use the technique of Mittag-Leffler sequence. Since $\Omega_{\varepsilon_{n}}$ is a Runge domain, $h_{n}$ can be approximated by entire functions; thus, there is an entire function $h_{n}^{\prime}$ so that

$$
\left\|h_{n}-h_{n}^{\prime}\right\|_{L^{\infty}\left(\Omega_{\varepsilon_{n}}\right)}<1 / 2^{n}
$$

Set

$$
f=f_{1}+\sum_{n=1}^{\infty}\left(f_{n+1}-f_{n}-h_{n}^{\prime}\right) .
$$

Note that $f=f_{j}-h_{1}^{\prime}-\cdots-h_{j-1}^{\prime}+\sum_{n=j}^{\infty}\left(f_{n+1}-f_{n}-h_{n}^{\prime}\right)$. It is in fact well defined on $\Omega \backslash D_{2}$ and solves the equation $\bar{\partial} f=g$.

As we know, Greiner-Kohn-Stein condition (4.2) is closely tied to the problem of solving $\bar{\partial}$ equation with compact support. As an analog, condition (6.4) is related to solving a $\bar{\partial}$ equation with partial compact support in $\Omega_{1}=D_{2}+i \mathbf{R}^{2}$. Namely, given a smooth $(0,2)$ form $G$ in $\Omega_{1}$ with compact support in radial direction $\left(G=0\right.$ outside of $\Omega_{R}=D_{2}+i R D_{2}$ for some large $\left.R>0\right)$, when can one find a smooth $(0,1) g$ also with compact support in radial direction so that $\bar{\partial} g=G$ ?

Proposition 6.3.1. The above problem has a solution if and only if the analytic functional defined by $\psi \mapsto \int G \psi$ is carried by $E_{R}=\partial D_{2}+i R D_{2}$ for some $R>0$.

Proof. First, we can always find a smooth $g_{1}$ so that $\bar{\partial} g_{1}=G$. Notice that $G$ can be solved with compact support in radial direction if and only if $g_{1}$ is $\bar{\partial}$ exact in radial direction. By Lemma 6.3.1, $g_{1}$ is $\bar{\partial}$ exact outside of $\Omega_{R}$ for some $R>0$ if and only if the analytic functional $\psi \mapsto \int_{S_{\delta}} g_{1} \psi$ is carried by $S_{\delta}^{\prime}$ with $X=\Omega_{R}$. Then, Stokes' formula implies the conclusion of the lemma.

Finally, we remark that any $\bar{\partial}$ closed smooth form in $D_{2}+i \widetilde{D}_{2}$ can be extended to $C^{2} \backslash D_{2}$ as a $\bar{\partial}$ closed form. This shows that the Tsuno forms discussed in $\S 4.1$ is basically equivalent to the $\bar{\partial}$ closed form defined in a cylindrical domain.

\section{APPENDIX. AN ELEMENTARY PROOF (AND EXTENSION) OF PINCUK'S EDGE-OF-THE-WEDGE THEOREM FOR DISTRIBUTIONS}

A.1. An estimate for the Baouendi-Treves approximation. In this section, we give a pointwise estimate for the Baouendi-Treves approximation scheme [BT, T] on a totally real manifold, which will be used to prove Pinčuk's edge-of-thewedge theorem in the next section. An extension of Pinčuk's theorem is also 
provided. $M$ is assumed to be $C^{\infty}$ smooth. We also inherit the notations from $\S 6.2$. Let $f$ be a holomorphic function defined in $W_{\rho}(\Gamma)$ and satisfies $(6.1 \mathrm{k})$. And let $\chi \in C_{0}^{\infty}\left(M_{\rho}\right)$ be a cut-off function which is identically 1 in a neigborhood of 0 and satisfies

$$
\sup _{x \in \operatorname{supp} \chi}\left|\phi^{\prime}(x)\right|<1 / 2 .
$$

Then, we define the Baouendi-Treves approximation scheme

$$
T_{\tau} f(z)=\left\langle B f,(\tau / 2 \pi)^{m / 2} e^{-\tau[z-\cdot]^{2}} \chi(\cdot)\right\rangle .
$$

The following is our estimates.

Proposition A.1.1. Under the above assumption, there are positive constants $c_{1}$, $c_{2}, C$ and a neighborhood $U$ of 0 , which are independent of $f, \tau$, such that $\forall z \in W_{\rho}(\Gamma) \cap U$,

(1) $\left|T_{\tau} f(z)\right| \leq C[\operatorname{dist}(z, M)]^{-k}$;

(2)

$$
\begin{aligned}
\left|T_{\tau} f(z)-f(z)\right| \leq & C \max _{\zeta \in O_{x, \delta}}|f(z)-f(Z(\zeta)+i(y-\varphi(x)))| \\
& +\frac{C e^{-c_{1} \delta^{2} \tau}}{[\operatorname{dist}(z, M)]^{k}}+C e^{-c_{2} \tau},
\end{aligned}
$$

where $x=\operatorname{Re} z, y=\operatorname{Im} z, O_{x, \delta}$ is the $\delta$-ball in $\mathbf{R}^{m}$ with center $x$.

The proof of the proposition is basically a standard approximation estimate with the help of the following well-known lemma and the technique of integration by parts.

Lemma A.1.1. If $f$ is a function satisfying (6.2k) and $|\bar{\partial} f| \leq C$ in $W_{\rho}(\Gamma)$, then there is a bounded holomorphic function $F(f)$ in $W_{\rho}(\Gamma)$ satisfying

$$
\partial^{k+1} F(f) / \partial z_{1}^{k+1}=f .
$$

The proofs will be omitted. As an easy consequence, we see that $T_{\tau} f$ converges to $f$ pointwise in $W_{\rho}(\Gamma)$.

A.2. Proof of Pinčuk's theorem. As we know, the edge-of-the-wedge theorem for distributions can be proved either by a cohomology method [P] or by FBI transform [BCT, BR1]. Here, we give a much more elementary proof based on Baouendi-Treves approximation technique.

Theorem A.2.1. If $f$ is holomorphic in $W_{\rho}(\Gamma) \cup W_{\rho}(-\Gamma)$ and $B f_{\Gamma}=B f_{-\Gamma}$ in the distribution sense, then $f$ can be holomorphically extended to a neighborhood of 0 .

Proof. We actually prove the following statement:

$T_{\tau} f \rightarrow \tilde{f}$ uniformly in a neighborhood of 0 as $\tau \rightarrow+\infty$ with $\tilde{f}$ coincided with $f$ on $W_{\rho}(\Gamma) \cup W_{\rho}(-\Gamma)$.

Then, we see that $\tilde{f}$ serves as a holomorphic extension of $f$ to a neighborhood of 0 . To prove the above statement, we need a family of polynomial disks designed by Rosay [Ro]. Choose $X_{0} \in T_{0} M=\mathbf{R}^{m}$, where $T_{0} M$ is the 
tangent space of $M$ at 0 , such that there are real numbers $a$ and $b$ satisfying $a X_{0}+b\left(i X_{0}\right) \in W_{\rho}(\Gamma)$. Define

$$
\varphi_{w}^{\varepsilon}: z \in \Delta^{1} \mapsto \varphi_{w}^{\varepsilon}(z)=z\left(\varepsilon X_{0}\right)+\alpha(\varepsilon) \frac{z(z+1)}{2}+\beta(\varepsilon) \frac{z(z-1)}{2}-\varepsilon^{2} w\left(z^{2}-1\right),
$$

where $\alpha(\varepsilon), \beta(\varepsilon)$ are so chosen that $\varepsilon X_{0}+\alpha(\varepsilon) \in M,-\varepsilon X_{0}+\beta(\varepsilon) \in M$ and $\alpha(\varepsilon)=o(\varepsilon), \beta(\varepsilon)=o(\varepsilon)$ as $\varepsilon \rightarrow 0, w \in \mathbf{C}^{m},|w| \leq 1$. Then, $\varphi_{w}^{\varepsilon}$ enjoys the following properties.

Lemma A.2.1. (a) For all $\theta \in(0, \pi), \varphi_{w}^{\varepsilon}\left(e^{i \theta}\right) \in W_{\rho}(\Gamma)$, and for all $\theta \in$ $(-\pi, 0), \varphi_{w}^{\varepsilon}\left(e^{i \theta}\right) \in W_{\rho}(-\Gamma), \varphi_{w}^{\varepsilon}(1), \varphi_{w}^{\varepsilon}(-1) \in M$.

(b) $\varphi_{w}^{\varepsilon}(0)=\varepsilon^{2} w$.

(c) $\operatorname{dist}\left(\varphi_{w}^{\varepsilon}\left(e^{i \theta}\right), E\right) \geq C \varepsilon|\sin \theta|$,

where $C>0$ is independent of $\varepsilon, w$ ( $\varepsilon$ small, $|w| \leq 1)$.

The proof of the lemma will be given later. Let us continue the proof of the theorem. Let $z \in \Delta^{1}$. The main trick in our proof is to introduce

$$
\widetilde{T}_{\tau}^{\varepsilon, w} f(z)=:\left(z^{2}-1\right)^{k+1} T_{\tau} f\left(\varphi_{w}^{\varepsilon}(z)\right),
$$

which suppresses the growth of $T_{\tau} f$ near the edge, while it keeps the center of the disk invariant (except for a signature). Suppose that $\varepsilon$ is small enough that $\varphi_{w}^{\varepsilon}(z) \in U, \forall z \in \Delta^{1},|w| \leq 1$. Then, from Proposition A.1.1 and Lemma A.2.1, it is elementary to show the following statement. On the boundary $b \Delta^{1}$ of $\Delta^{1}, \widetilde{T}_{\tau}^{\varepsilon, w} f(z)$ converges to $\left(z^{2}-1\right)^{k+1} f\left(\varphi_{w}^{\varepsilon}(z)\right)$ uniformly for $z \in b \Delta^{1}$, $|w| \leq 1$ as $\tau \rightarrow+\infty$. Here, $\varepsilon$ is a fixed small positive number.

Since $\widetilde{T}_{\tau}^{\varepsilon, w} f(z)$ is uniformly convergent for $z \in b \Delta^{1},|w| \leq 1$ and holomorphic in the unit disk, it follows that

$$
\widetilde{T}_{\tau}^{\varepsilon, w} f(z) \rightarrow \text { some function } f_{w}^{\varepsilon}(z)
$$

uniformly for $z \in \Delta^{1},|w| \leq 1$ as $\tau \rightarrow+\infty$. As a consequence,

$$
T_{\tau} f\left(\varepsilon^{2} w\right)=T_{\tau} f\left(\varphi_{w}^{\varepsilon}(0)\right)=(-1)^{k+1} \widetilde{T}_{\tau}^{\varepsilon, w} f(0) \rightarrow(-1)^{k+1} f_{w}^{\varepsilon}(0)
$$

uniformly for $|w| \leq 1$. Note that $T_{\tau} f(z) \rightarrow f(z)$ pointwise for $z \in W_{\rho}(\Gamma) \cup$ $W_{\rho}(-\Gamma)$ by Proposition A.1.1. Therefore, in the $\varepsilon^{2}$-ball of $\mathbf{C}^{m}, T_{\tau} f(z) \rightarrow \tilde{f}(z)$ uniformly which coincides with $f(z)$, for all $z \in W_{\rho}(\Gamma) \cup W_{\rho}(-\Gamma)$. We finish the proof of the theorem.

Proof of Lemmma A.2.1. (a) and (b) were proved in Rosay [Ro]. Since the proof is simple, for convenience of reference, we copy it down here. In fact, one notices that there exists $r>0$ so that for all $e \in M,|e|<r, X \in \mathbf{C}^{m}$, $\left|X-X_{0}\right|<r$, and $\lambda \in \mathbf{C},|\arg \lambda| \leq \pi / 3$, one has

$$
e+\lambda(i X) \in W_{\rho}(\Gamma), \quad e-\lambda(i X) \in W_{\rho}(-\Gamma) .
$$

Obviously, $\varphi_{w}^{\varepsilon}(1), \varphi_{w}^{\varepsilon}(-1) \in M$. For $\theta \in[-\pi / 2, \pi / 2]$,

$$
\varphi_{w}^{\varepsilon}\left(e^{i \theta}\right)=\varphi_{w}^{\varepsilon}(1)+\int_{\left[1, e^{i \theta}\right]} \frac{\partial \varphi_{w}^{\varepsilon}}{\partial z} d z=\varphi_{w}^{\varepsilon}(1)+\left(\varepsilon i X_{0}+\sigma_{w}(\varepsilon)\right)\left(\frac{e^{i \theta}-1}{i}\right),
$$

where $\left|\sigma_{w}(\varepsilon)\right|=o(\varepsilon), \forall|w| \leq 1$. Note that $\left|\arg \left(\left(e^{i \theta}-1\right) / i\right)\right| \leq \pi / 4$. For small $\varepsilon$ (independent of the choice of $w),(\mathrm{A} .1)$ yields $\varphi_{w}^{\varepsilon}\left(e^{i \theta}\right) \in W_{\rho}(\Gamma)$, if $\theta \in(0, \pi / 2]$, and $\varphi_{w}^{\varepsilon}\left(e^{i \theta}\right) \in W_{\rho}(-\Gamma)$, if $\theta \in[-\pi / 2,0)$. For the other $\theta$, we 
choose $\varphi_{w}^{\varepsilon}(-1)$ instead of $\varphi_{w}^{\varepsilon}(1)$ as our starting point. We show part (c) next. Note that

$$
\frac{d \varphi_{w}^{\varepsilon}\left(e^{i \theta}\right)}{d \theta}=i e^{i \theta}\left(\varepsilon X_{0}\right)+o(\varepsilon) X_{w}(\theta)=\varepsilon e^{i \theta}\left(i X_{0}+\frac{o(\varepsilon)}{\varepsilon} X_{w}(\theta)\right)
$$

where $\left|X_{w}\right| \leq C$ uniformly for $|w| \leq 1$. Hence, when $\varphi_{w}^{\varepsilon}\left(e^{i \theta}\right)$ intersects with $M$, i.e., $\theta=0, \pi$, the tangent direction is in a very small conic neighborhood of $i X_{0}$, which means the curve $\varphi_{w}^{\varepsilon}\left(e^{i \theta}\right)$ is almost perpendicular to the edge $M$, for small $\varepsilon,|w| \leq 1$. Therefore, the distance between $\varphi_{w}^{\varepsilon}\left(e^{i \theta}\right)$ and $M$ is almost the distance between $\varphi_{w}^{\varepsilon}\left(e^{i \theta}\right)$ and its intersection with $M$, which means that there is a constant $C>0$ independent of $\varepsilon, w$ such that

$$
\operatorname{dist}\left(\varphi_{w}^{\varepsilon}\left(e^{i \theta}\right), M\right) \geq C \cdot \operatorname{dist}\left(\varphi_{w}^{\varepsilon}\left(e^{i \theta}\right),\left\{\varphi_{w}^{\varepsilon}(1), \varphi_{w}^{\varepsilon}(-1)\right\}\right) .
$$

For $\theta \in[-\pi / 2, \pi / 2]$,

$$
\begin{aligned}
\left|\varphi_{w}^{\varepsilon}\left(e^{i \theta}\right)-\varphi_{w}^{\varepsilon}(1)\right|= & \mid\left(e^{i \theta}-1\right)\left(\varepsilon X_{0}\right)+\alpha(\varepsilon) \frac{\left(e^{i \theta}-1\right)\left(e^{i \theta}+2\right)}{2} \\
& \quad+\beta(\varepsilon) \frac{e^{i \theta}\left(e^{i \theta}-1\right)}{2}-\varepsilon^{2} w\left(e^{i \theta}-1\right)\left(e^{i \theta}+1\right) \mid . \\
= & \varepsilon\left|e^{i \theta}-1\right| \cdot\left|X_{0}+o(1)\right|,
\end{aligned}
$$

where, $o(1) \rightarrow 0$ uniformly as $\varepsilon \rightarrow 0$. When $\varepsilon$ is small, $\left|X_{0}+o(1)\right| \geq\left|X_{0}\right| / 2$. Therefore,

$$
\operatorname{dist}\left(\varphi_{w}^{\varepsilon}\left(e^{i \theta}\right), M\right) \geq C\left|\varphi_{w}^{\varepsilon}\left(e^{i \theta}\right)-\varphi_{w}^{\varepsilon}(1)\right| \geq C \varepsilon\left|e^{i \theta}-1\right| \geq C \varepsilon|\sin \theta| .
$$

For other $\theta$, by considering $\varphi_{w}^{\varepsilon}(-1)$ instead of $\varphi_{w}^{\varepsilon}(1)$, we can obtain the estimate in the same way.

It is worth noticing that when $M$ is only $C^{1}$ smooth, while $f$ still has polynomial growth near the edge, the hyperfunction boundary value $B f_{\Gamma}^{X}$ serves as a natural extension of the distribution boundary value. More importantly, following the above proof (we use the approximation scheme $u_{\tau}$ defined in (3.4) instead of $T_{\tau} f$; then, Proposition A.1.1 is trivial for $u_{\tau}$ and the argument in the above proof of Pinčuk's theorem can still be used), we can generalize Pinčuk's edge-of-the-wedge theorem to the case of a $C^{1}$ edge. Namely, we have the following proposition.

Proposition A.2.1. Suppose that $M$ is $C^{1}$ smooth, and $f$ is holomorphic in $W_{\rho}(\Gamma) \cup W_{\rho}(-\Gamma)$ and has polynomial growth near the edge $M$. If $B f_{\Gamma}^{X}=B f_{-\Gamma}^{X}$ for some neighborhood $X$ of 0 , then $f$ can be holomorphically extended to a neighborhood of 0 .

This proposition also serves as a trivial generalization of the continuous version of the edge-of-the-wedge theorem with $C^{1}$ edge in [Ro]. One could have noticed that there is still a gap between the above generalization and the hyperfunction version of the edge-of-the-wedge theorem given in $\S 5.3$.

\section{ACKNOWLEDGMENT}

Since this is adapted from my Ph.D. thesis at the University of Wisconsin at Madison, I wish to express my deep gratitude to my thesis advisor Professor 
Jean-Pierre Rosay for his many inspiring suggestions, his constant encouragement and various help. I am also grateful to Professors Alex Nagel and Walter Rudin for their useful suggestions and financial support. Finally, thanks go to Professors Baouendi and Rothschild for helpful remarks, as well as Professor R. Harvey for showing me his paper. The revision of the paper was finished when the author was visiting the Department of Mathematics of the University of California-San Diego. It is also a pleasure to thank the organization for providing such a nice working environment.

\section{REFERENCES}

[Al] H. Alexander, Personal communication to Rosay and Stout.

[Am] E. Amar, On the extension of C.R. functions, preprint.

[AH] A. Andreotti and D. C. Hill, E. E. Levi convexity and the Hans Lewy problem. I, II, Ann. Scuola Norm. Sup. Pisa 26 (1972), 325-363, 747-806.

[BCT] M. S. Baouendi, C. H. Chang, and F. Treves, Microlocal hypo-analyticity and extension of CR functions, J. Differential Geom. 18 (1983), 331-391.

[BR1] M. S. Baouendi and L. Rothschild, Normal forms for generic manifolds and holomorphic extension of CR functions, J. Differential Geom. 25 (1987), 431-467.

[BR2] _ Extension of holomorphic functions in generic wedges and their wave front sets, Comm. Partial Differential Equations 13 (1988), 1441-1466.

[BRT] M. S. Baouendi, L. Rothschild, and F. Treves, CR structure with group action and extendability of CR functions, Invent. Math. 82 (1985), 359-396.

[BT] M. S. Baouendi and F. Treves, A property of the functions and distributions annihilated by a locally integrable system of complex vector fields, Ann. of Math. (2) 113 (1981), 387-421.

[Cal] W. Calbeck, Ph.D. thesis, Univ. of Wisconsin-Madison, 1990.

[Dob] P. Dolbeault, Formes differentielles et cohomologie sur une variete analytique complexe. I, II, Ann. of Math. (2) 64 (1956), 83-130; 65 (1960) 94-123.

[Duf] A. Dufresnoy, Sur l'opérateur $d^{\prime \prime}$ et les fonctions différentiables au sens de Whitney, Ann. Inst. Fourier (Grenoble) 29 (1979), 229-238.

[Hv1] R. Harvey, The theory of hyperfunctions on totally real subsets of a complex manifold with applications to extension problems, Amer. J. Math. 91 (1969), 853-873.

[Hv2] __ Integral formulae connected by Dolbeault's isomorphism, Rice Univ. Stud. 51 (1970), 77-97.

[HvPo] R. Harvey and J. Polking, Fundamental solutions in complex analysis. I, II, Duke Math. J. 46 (1979), 253-340.

[HvWe] R. Harvey and R. O. Wells, Holomorphic approximation and hyperfunction theory on a $C^{1}$ totally real submanifold of a complex manifold, Math. Ann. 197 (1972), 287-318.

[HL] G. M. Henkin and J. Leiterer, Theory of functions on complex manifolds, Birkhäuser, Boston, Mass., 1984.

[H1] L. Hörmander, The analysis of linear partial differential operators. I, Springer-Verlag, Berlin, Heidelberg, and New York, 1983.

[H2] $\_, L^{2}$ estimates and existence theorems for the $\bar{\partial}$ operator, Acta Math. 113 (1965), 89-152.

[M1] A. Martineau, Le "edge of the wedge theorem" en théorie des hyperfonctions de Sato, Proc. Int. Conf. Funct. Anal. Rel. Topics, Univ. of Tokyo Press, Tokyo, 1970, pp. 95-106.

[M2] _ Sur les functionelles analytiques et la transformation de Fourier-Borel, J. Analyse Math. 11 (1963), 1-164.

[M3] Distributions et valeurs au bord des fonctions holomorphes, Theory of Distributions (Proc. Internat. Inst., Lisbon, 1964), Inst. Gulbenkian Cc., Lisbon, 1964, pp. 193-326.

[P] S. Pinčuk, Bogoliubov's theorem on the "edge of the wedge" for generic manifolds, Math USSR-Sb. 23 (1974), 441-455. 
[RSi] M. Range and Y. T. Siu, Uniform estimates for the $\bar{\partial}$ equation on domains with piecewise smooth strictly pseudoconvex boundaries, Math. Ann. 206 (1973), 325-354.

[Ro] J.-P. Rosay, A propos de "wedges" et d' "edges" et de prolongements holomorphes, Trans. Amer. Math. Soc. 297 (1986), 63-72.

[RoSt] J.-P. Rosay and E. L. Stout, Rado's theorem for CR-functions, preprint.

[Ru] W. Rudin, Lectures on the edge of the wedges theorem, CBMS Regional Conf. Ser. in Math., no. 6, Amer. Math. Soc., Providence, R.I., 1971.

[S] M. Sato, Theory of hyperfunctions. I, J. Fac. Sci. Univ. Tokyo I 8 (1959), 139-193.

[SKK] M. Sato, T. Kawai, and M. Kashiwara, Microfunctions and pseudo-differential equations, Lecture Notes in Math., vol. 287, Springer-Verlag, Berlin, 1973, pp. 265-529.

[Sch] P. Schapira, Théorie des hyperfonctions, Lecture Notes in Math., vol. 126, Springer-Verlag, Berlin and New York, 1970.

[Ser] J.-P. Serre, Une theoreme de dualite, Comment. Math. Helv. 29 (1955), 9-26.

[T] F. Tréves, Approximation and representation of functions and distributions annihilated by a system of complex vector fields, Ecole Polytechnique (1981).

[Ts] Y. Tsuno, Integral representation of an analytic functional, J. Math. Soc. Japan 34 (1982), 379-391.

Department of Mathematics, University of Michigan, Ann Arbor, Michigan 481091003

E-mail address: zye@math.lsa.umich.edu 